\title{
FULLY COMPUTABLE A POSTERIORI ERROR BOUNDS FOR HYBRIDIZABLE DISCONTINUOUS GALERKIN FINITE ELEMENT APPROXIMATIONS
}

\author{
MARK AINSWORTH AND GUOSHENG FU
}

\begin{abstract}
We derive a posteriori error estimates for the hybridizable discontinuous Galerkin (HDG) methods, including both the primal and mixed formulations, for the approximation of a linear second-order elliptic problem on conforming simplicial meshes in two and three dimensions.

We obtain fully computable, constant free, a posteriori error bounds on the broken energy seminorm and the HDG energy (semi)norm of the error. The estimators are also shown to provide local lower bounds for the HDG energy (semi)norm of the error up to a constant and a higher-order data oscillation term. For the primal HDG methods and mixed HDG methods with an appropriate choice of stabilization parameter, the estimators are also shown to provide a lower bound for the broken energy seminorm of the error up to a constant and a higher-order data oscillation term. Numerical examples are given illustrating the theoretical results.
\end{abstract}

\section{INTRODUCTION}

Recent years have seen the developments of fully computable, guaranteed error bounds for the conforming $6,7,14,15,18,30,37,40,42,43,51$, nonconforming [1, 8 , 19, 29, 33, 38, 39, discontinuous Galerkin $2,9,9,10,21,38,39$, and mixed finite element methods [3, 5, 27, 38]; see also unified frameworks in 4, 16, 34].

In comparison, there are relatively few works on a posteriori error estimates for the hybridizable discontinuous Galerkin (HDG) methods 22. The a posteriori estimates for HDG methods that are currently available in the literature 17, 20, 25, 26, 31, 35] are all of residual type, in which reliability is shown up to a generic (unknown) constant. This means that, while the associated estimation may be suitable as local refinement indicators, they cannot provide a quantitative stopping criterion. Moreover, if only a single fixed mesh is used (as is often the case in practice) then the value of an a posteriori bound containing unknown constants is somewhat questionable. Here we present, for the first time, fully computable a posteriori error bounds for HDG methods, for both the primal and mixed formulations, in the setting of a linear second-order elliptic problem on conforming simplicial meshes in two and three space dimensions. The key ingredient of our analysis is the local conservation property of the HDG methods, with which cheap element-wise equilibrated fluxes can be constructed.

1991 Mathematics Subject Classification. 65N30. 65Y20. 65D17. 68U07.

Key words and phrases. HDG, a posteriori error analysis, computable error bounds.

First author gratefully acknowledges the partial support of this work under AFOSR contract FA9550-12-1-0399. 
The remainder of this article is organized as follows. Section 2 presents the model problem and prepares the notation used throughout the article. In Section 3. we introduce the primal HDG schemes and the corresponding computation error bounds. While in Section 4, we introduce the mixed HDG schemes and the corresponding computation error bounds. Numerical results are then presented in Section 5. The proofs of the main results in Section 3 and Section 4 are presented in Section 6 .

\section{Preliminaries}

2.1. Model Problem. Consider the following model problem:

$$
\left.\begin{array}{rl}
\boldsymbol{\sigma}-a \nabla u & =0 \\
-\nabla \cdot \boldsymbol{\sigma} & =f \in L^{2}(\Omega)
\end{array}\right\} \text { in } \Omega
$$

subject to $u=0$ on $\partial \Omega$, where $\Omega \in \mathbb{R}^{d}, d \in\{2,3\}$, is a simply connected polygonal/polyhedral domain. The datum $a \in L^{\infty}(\Omega)$ is assumed to be strictly positive and, for simplicity, is assumed piecewise constant on subdomains of $\Omega$.

2.2. Notation and finite elements. We consider a family of partitions $\mathcal{T}_{h}=\{K\}$ of the domain $\Omega$ into the union of nonoverlapping, shape-regular, simplicial elements such that the nonempty intersection of a distinct pair of elements is a single common node, single common edge or single common face (in three dimensions). The family of partitions is assumed to be locally quasi-uniform in the sense that the ratio of the diameters of any pair of neighboring elements is uniformly bounded above and below over the whole family. Furthermore, it is assumed that the partitioning is compatible with the data so that $a$ is constant on each element.

The set of all facets (edges in two dimensions and faces in three dimensions) of the elements is denoted by $\mathcal{E}_{h}$, which we partition into subsets $\mathcal{E}_{h}^{\partial}$ and $\mathcal{E}_{h}^{o}$ consisting of facets lying on the boundary $\partial \Omega$, and the remaining interior facets, respectively. Likewise, the corresponding quantities relative to an individual element $K$ are denoted by $\mathcal{E}(K), \mathcal{E}^{\partial}(K)$, and $\mathcal{E}^{o}(K)$, respectively. For each facet $F \in \mathcal{E}_{h}$, the set $\widetilde{F}$ consists of those elements for which $F$ is a facet,

$$
\widetilde{F}=\left\{K^{\prime} \in \mathcal{T}_{h}: F \in \mathcal{E}\left(K^{\prime}\right)\right\},
$$

while, for each element $K \in \mathcal{T}_{h}$, the set $\widetilde{K}$ consists of those elements having a facet in common with $K$,

$$
\widetilde{K}=\left\{K^{\prime} \in \mathcal{T}_{h}: \mathcal{E}(K) \cap \mathcal{E}\left(K^{\prime}\right) \text { is nonempty }\right\} .
$$

Let $h_{D}$ denote the diameter of a domain $D,|K|$ denote the measure of an element $K,|F|$ denote the measure of a facet $F$, and $|\partial K|=\sum_{F \in \mathcal{E}(K)}|F|$ denote the measure of the boundary of an element $K$.

Let $\Sigma_{h, k}, V_{h, k}$, and $M_{h, k}$ denote the finite dimensional spaces

$$
\begin{aligned}
\Sigma_{h, k}:=\left\{\boldsymbol{\tau} \in L^{2}\left(\mathcal{T}_{h}\right)^{d}:\right. & \left.\left.\boldsymbol{\tau}\right|_{K} \in \mathbb{P}_{k}(K)^{d} \quad \forall K \in \mathcal{T}_{h}\right\}, \\
V_{h, k}:=\left\{v \in L^{2}\left(\mathcal{T}_{h}\right):\right. & \left.\left.v\right|_{K} \in \mathbb{P}_{k}(K) \forall K \in \mathcal{T}_{h}\right\}, \\
M_{h, k}:=\left\{\widehat{v} \in L^{2}\left(\mathcal{E}_{h}\right):\right. & \left.\left.\widehat{v}\right|_{F} \in \mathbb{P}_{k}(F) \forall F \in \mathcal{E}_{h}\right\},
\end{aligned}
$$

where $\mathbb{P}_{k}(D)$ denotes the set of polynomials of degree at most $k \geq 0$ on the domain $D$. The space of homogeneous polynomials of degree $k$ on a domain $D$ is denoted 
as $\widetilde{\mathbb{P}}_{k}(D)$. We shall also need the subspace $M_{h, k}^{0} \subset M_{h, k}$ given by

$$
M_{h, k}^{0}:=\left\{\widehat{v} \in M_{h, k}:\left.\widehat{v}\right|_{F}=0 \forall F \in \mathcal{E}_{h}^{\partial}\right\} .
$$

To simplify notation, we introduce the compound finite-dimensional space

$$
\mathbb{V}_{h, k, \delta}:=V_{h, k} \times M_{h, k-\delta}^{0}, \quad k \geq 1, \delta \in\{0,1\},
$$

which is used for the primal HDG scheme, while the compound finite-dimensional space

$$
\mathbb{X}_{h, k}:=\Sigma_{h, k} \times V_{h, k} \times M_{h, k}^{0}, \quad k \geq 0,
$$

is used for the mixed HDG scheme.

The stabilization parameters for the HDG schemes will be taken from the space $M_{h, 0}^{\mathrm{dc}}$, where, for any nonnegative integer $m$,

$$
M_{h, m}^{\mathrm{dc}}:=\Pi_{K \in \mathcal{T}_{h}} \mathbb{P}_{m}(\partial K),
$$

with $\mathbb{P}_{m}(\partial K):=\left\{\mu \in L^{2}(\partial K):\left.\mu\right|_{F} \in \mathbb{P}_{m}(F) \forall F \in \mathcal{E}(K)\right\}$.

We use the standard notation for jumps and averages 12 of functions in $M_{h, m}^{\mathrm{dc}}$ and $\left[M_{h, m}^{\mathrm{dc}}\right]^{d}$ on the mesh skeleton $\mathcal{E}_{h}$ : Let $F$ be a facet shared by elements $K^{+}$and $K^{-}$with unit normal vectors $\boldsymbol{n}^{+}$and $\boldsymbol{n}^{-}$on $F$ pointing exterior to $K^{+}$and $K^{-}$ respectively, then for $q \in M_{h, m}^{\mathrm{dc}}$

$$
\{q\}=\frac{1}{2}\left(\left.q\right|_{K^{+}}+\left.q\right|_{K^{-}}\right), \quad \llbracket q \rrbracket=\left.q\right|_{K^{+}} \boldsymbol{n}^{+}+\left.q\right|_{K^{-}} \boldsymbol{n}^{-} \quad \text { on } F \in \mathcal{E}_{h}^{o} .
$$

Similarly, for $\phi \in\left[M_{h, m}^{\mathrm{dc}}\right]^{d}$ we set

$$
\{\phi\}=\frac{1}{2}\left(\left.\phi\right|_{K^{+}}+\left.\phi\right|_{K^{-}}\right), \quad \llbracket \phi \rrbracket=\left.\phi\right|_{K^{+}} \cdot \boldsymbol{n}^{+}+\left.\phi\right|_{K^{-}} \cdot \boldsymbol{n}^{-} \quad \text { on } F \in \mathcal{E}_{h^{o}}^{o}
$$

On a boundary facet $F \in \mathcal{E}_{h}^{\partial}$, for each $q \in M_{h, m}^{\mathrm{dc}}$ we set

$$
\{q\}=q, \quad \llbracket q \rrbracket=q \boldsymbol{n} \quad \text { on } F \in \mathcal{E}_{h}^{\partial} .
$$

We use the notation $(\cdot, \cdot)_{\omega}$ to denote the integral inner product over a region $\omega \subset \mathbb{R}^{d}$, and $\|\cdot\|_{\omega}$ to denote the corresponding $L^{2}$-norm. We omit the subscript in the case when $\omega$ is the physical domain $\Omega$. Finally, for each element $K \in \mathcal{T}_{h}$, we use the notation $\langle\cdot, \cdot\rangle_{\partial K}$ to denote the integral inner product over the element boundary $\partial K$.

\section{The PRimal HDG methods AND COMPutable ERROR BOUnds}

3.1. Primal HDG formulation. Let $\alpha_{h} \in M_{h, 0}^{\mathrm{dc}}$ be a positive stabilization parameter to be specified later, and define the bilinear form $\mathcal{B}_{h}^{p r}: \mathbb{V}_{h, k, \delta} \times \mathbb{V}_{h, k, \delta} \rightarrow \mathbb{R}$ by

$$
\begin{array}{r}
\mathcal{B}_{h}^{p r}((u, \widehat{u}),(v, \widehat{v}))=\sum_{K \in \mathcal{T}_{h}}\left\{(a \nabla u, \nabla v)_{K}-\langle a \nabla u \cdot \boldsymbol{n}, v-\widehat{v}\rangle_{\partial K}\right. \\
\left.-\langle a \nabla v \cdot \boldsymbol{n}, u-\widehat{u}\rangle_{\partial K}+\left\langle\alpha_{h}\left(P_{M} u-\widehat{u}\right), P_{M} v-\widehat{v}\right\rangle_{\partial K}\right\},
\end{array}
$$

where $P_{M}$ denotes the $L^{2}$ projection onto the space $M_{h, k-\delta}^{\mathrm{dc}}$. We also define the linear form $\mathcal{L}_{h}^{p r}: \mathbb{V}_{h, k, \delta} \rightarrow \mathbb{R}$ by

$$
\mathcal{L}_{h}^{p r}((v, \widehat{v}))=\sum_{K \in \mathcal{T}_{h}}(f, v)_{K} .
$$


Let $u$ be the solution of (1). An approximation of $\left(u,\left.u\right|_{\mathcal{E}_{h}}\right)$ is obtained by seeking $\left(u_{h}, \widehat{u}_{h}\right) \in \mathbb{V}_{h, k, \delta}$ such that

$$
\mathcal{B}_{h}^{p r}\left(\left(u_{h}, \widehat{u}_{h}\right),\left(v_{h}, \widehat{v}_{h}\right)\right)=\mathcal{L}_{h}^{p r}\left(\left(v_{h}, \widehat{v}_{h}\right)\right) \quad \forall\left(v_{h}, \widehat{v}_{h}\right) \in \mathbb{V}_{h, k, \delta} .
$$

This scheme is known as the hybridized, symmetric, interior penalty discontinuous Galerkin method 41 .

3.2. The choice of the stabilization parameter $\alpha_{h}$. It is well-known 41] that (11) is well-posed provided the stabilization parameter is "sufficiently large". The following result quantifies exactly how large $\alpha_{h}$ must be; similar results for interior penalty discontinuous Galerkin methods can be found in $2,9,11,32,48]$.

Lemma 1. Suppose the stabilization parameter $\alpha_{h}$ is given by

$$
\left.\alpha_{h}\right|_{F}=\frac{\left.a\right|_{K} \gamma}{|F|} \sum_{F^{\prime} \in \mathcal{E}(K)} \frac{\left|F^{\prime}\right|^{2}}{|K|} \quad \text { for all } F \in \mathcal{E}(K), \text { for all } K \in \mathcal{T}_{h},
$$

where $\gamma$ is a constant satisfying

$$
\gamma>\frac{k(k+d-1)}{d} .
$$

Then (11) has a unique solution $\left(u_{h}, \widehat{u}_{h}\right) \in \mathbb{V}_{h, k, \delta}$ for $k \geq 1$ and $\delta \in\{0,1\}$.

The proof of this and other results in this section is postponed to Section 6

Remark 1. Observe that the stabilization parameter (12) on a facet $F$ is proportional to $h_{F}^{-1}$, which gives an optimal order a priori convergence rate $\mathcal{O}\left(h^{k}\right)$ in the energy norm [41, 44].

3.3. The broken energy seminorm and the HDG energy norm. For a given function $(v, \widehat{v}) \in \mathbb{V}_{h, k, \delta}+\left(H^{1}(\Omega) \times L^{2}\left(\mathcal{E}_{h}\right)\right)$, let the broken energy seminorm $\|\cdot\| \|$ be denoted by

$$
\|(v, \widehat{v})\|_{p r}=\left(\sum_{K \in \mathcal{T}_{h}}(a \nabla v, \nabla v)_{K}\right)^{1 / 2} .
$$

Our objective is to derive a fully computable estimator for the error in the HDG finite-element approximation $\left(e_{u}, \widehat{e}_{u}\right)=\left(u-u_{h},\left.u\right|_{\mathcal{E}_{h}}-\widehat{u}_{h}\right)$, where $u$ is the solution to (1) and $\left(u_{h}, \widehat{u}_{h}\right)$ is the solution to (11). Let the HDG energy norm $\|\cdot \cdot\|_{H D G, p r}$ be denoted by

$$
\|(v, \widehat{v})\|_{H D G, p r}=\left(\|(v, \widehat{v})\|_{p r}^{2}+\sum_{K \in \mathcal{T}_{h}}\left\langle\alpha_{h} P_{M}(v-\widehat{v}), P_{M}(v-\widehat{v})\right\rangle_{\partial K}\right)^{1 / 2} .
$$

Observe that, since $P_{M}\left(e_{u}-\widehat{e}_{u}\right)=-P_{M}\left(u_{h}-\widehat{u}_{h}\right)$, the quantity

$$
\sum_{K \in \mathcal{T}_{h}}\left\langle\alpha_{h} P_{M}\left(e_{u}-\widehat{e}_{u}\right), P_{M}\left(e_{u}-\widehat{e}_{u}\right)\right\rangle_{\partial K}
$$

is directly computable in terms of the HDG approximation $\left(u_{h}, \widehat{u}_{h}\right)$. Hence, given a constant free estimator for $\left\|\left(e_{u}, \widehat{e}_{u}\right)\right\|_{p r}$, we automatically have a constant free estimator for the HDG energy norm of the error as well. The next result shows that, by analogy with the standard interior penalty methods [2,10], these norms are equivalent in the following sense. 
Lemma 2. Let the stabilization parameter be given by (12) with the global constant $\gamma$ satisfying (13), then the HDG energy norm and the broken energy seminorm of the error $\left(e_{u}, \widehat{e}_{u}\right)=\left(u-u_{h},\left.u\right|_{\mathcal{E}_{h}}-\widehat{u}_{h}\right)$ are equivalent. That is to say,

$$
\left\|\left(e_{u}, \widehat{e}_{u}\right)\right\|_{p r} \leq\left\|\left(e_{u}, \widehat{e}_{u}\right)\right\|_{H D G, p r},
$$

and there exists a positive constant $c$, depending only on the shape-regularity of the mesh, the polynomial degree $k$, and the local permeability ratio between neighboring elements, such that

$$
c\left\|\left(e_{u}, \widehat{e}_{u}\right)\right\|_{H D G, p r}^{2} \leq\left\|\left(e_{u}, \widehat{e}_{u}\right)\right\|_{p r}^{2}+\sum_{K \in \mathcal{T}_{h}} o s c_{k-1}^{2}(f, K) .
$$

where the data oscillation on an element $K \in \mathcal{T}_{h}$ is defined to be

$$
o s c_{m}(f, K)=a^{-1 / 2} h_{K} \inf _{p \in \mathbb{P}_{m}}\|f-p\|_{K} .
$$

The proof of this result is postponed to Section 6 .

3.4. Local conservation. The numerical flux is defined by

$$
\widehat{\boldsymbol{\sigma}}_{h, p r}:=a \nabla u_{h}-\alpha_{h}\left(P_{M} u_{h}-\widehat{u}_{h}\right) \boldsymbol{n} \in\left[M_{h, k-\delta}^{\mathrm{dc}}\right]^{d}
$$

and satisfies the local conservation property

$$
(f, 1)_{K}+\left\langle\widehat{\boldsymbol{\sigma}}_{h, p r} \cdot \boldsymbol{n}, 1\right\rangle_{\partial K}=0 \quad \text { for each element } K \in \mathcal{T}_{h},
$$

along with

$$
\left.\llbracket \widehat{\boldsymbol{\sigma}}_{h, p r} \rrbracket\right|_{F}=0 \quad \text { on each interior facet } F \in \mathcal{E}_{h}^{o} .
$$

These results are a straightforward consequence of the definition and (11).

3.5. The computable error bounds. We obtain computable error bounds for the discrete energy norm of the error by bounding the conforming and non-conforming errors separately [2,4,10. To this end, two types of post-processing scheme will be needed.

3.5.1. Local (equilibrated) flux post-processing. We define a local flux post-processing [2, 9] as follows: Let $\boldsymbol{\sigma}_{h}^{*} \in \Sigma_{h, k}$ be such that, on each element $K$, there holds

$$
\begin{aligned}
\left(\nabla \cdot \boldsymbol{\sigma}_{h}^{*}, v\right)_{K} & =-(f, v)_{K} & & \forall v \in \mathbb{P}_{k-1}(K) \text { and }(v, 1)_{K}=0, \\
\left\langle\boldsymbol{\sigma}_{h}^{*} \cdot \boldsymbol{n}, \widehat{v}\right\rangle_{\partial K} & =\left\langle\widehat{\boldsymbol{\sigma}}_{h, p r} \cdot \boldsymbol{n}, \widehat{v}\right\rangle_{F} & & \forall \widehat{v} \in \mathbb{P}_{k}(F), \quad \forall F \in \mathcal{E}(K), \\
\left(\boldsymbol{\sigma}_{h}^{*}, \boldsymbol{\tau}\right)_{K} & =\left(a \nabla u_{h}, \boldsymbol{\tau}\right)_{K} & & \forall \boldsymbol{\tau} \in \Sigma_{k, s b b}(K),
\end{aligned}
$$

where

$$
\Sigma_{k, s b b}(K):=\left\{\boldsymbol{\tau} \in \mathbb{P}_{k}(K)^{d}: \nabla \cdot \boldsymbol{\tau}=0,\left.\boldsymbol{\tau} \cdot \boldsymbol{n}\right|_{F}=0 \text { for all } F \in \mathcal{E}(K)\right\}
$$

denotes the divergence-free "bubble" space. The unique solvability of 22 can be established using arguments similar to those used to study the closely related Brezzi-Douglas-Marini (BDM) projection [13. By the local conservation properties (20) and (21), we conclude that $\boldsymbol{\sigma}_{h}^{*} \in \Sigma_{h, k} \cap H($ div; $\Omega$ ) satisfies

$$
\nabla \cdot \boldsymbol{\sigma}_{h}^{*}=-\Pi_{k-1} f \text { on } \mathcal{T}_{h} \text {, and } \boldsymbol{\sigma}_{h}^{*} \cdot \boldsymbol{n}=\widehat{\boldsymbol{\sigma}}_{h, p r} \cdot \boldsymbol{n} \text { on } \partial \mathcal{T}_{h} .
$$

The quantity $\boldsymbol{\sigma}_{h}^{*}$ is usually referred to as an equilibrated flux 7 . 
3.5.2. Local potential post-processing. We obtain a globally continuous potential by a simple averaging 2, 2, 10] of the discontinuous potential $u_{h}$ as follows: Let $\mathcal{N}_{K, k}$ index a set of points $\left\{\boldsymbol{x}_{m}\right\}_{m \in \mathcal{N}_{K, k}}$ on $\bar{K}$ associated with a Lagrange basis for the conforming finite-element space of order $k$ on $\mathcal{T}_{h}$ and let $\mathcal{N}_{K, k}^{o}$ denote the restriction of the set $\mathcal{N}_{K, k}$ to the points that do not lie on the boundary of element $K$, with $\mathcal{N}_{\gamma, k}$ being its complementary set. Let $N_{\gamma, k}^{\partial}$ denote the restriction of the set $\mathcal{N}_{\gamma, k}$ to the points that lie on the closure of the boundary $\partial \Omega$. For $m \in \mathcal{N}_{\gamma, k}$, let $\Omega_{m}$ denote the set of elements in $\mathcal{T}_{h}$ whose closure contains the point $\boldsymbol{x}_{m}$.

The post-processed potential $u_{h}^{*} \in V_{h, k} \cap H^{1}(\Omega)$ is obtained through a simple averaging of the degrees of freedom for $u_{h}$ : for all elements $K \in \mathcal{T}_{h},\left.u_{h}^{*}\right|_{K}=$ $\left.S_{k}\left(u_{h}\right)\right|_{K} \in \mathbb{P}_{k}(K)$, where the nodal values are given by

$$
S_{k}\left(u_{h}\right)\left(\boldsymbol{x}_{m}\right)= \begin{cases}0 & \text { if } m \in \mathcal{N}_{\gamma, k}^{\partial}, \\ u_{h}\left(\boldsymbol{x}_{m}\right) & \text { if } m \in \mathcal{N}_{K, k}^{o}, \\ \left.\frac{1}{\# \Omega_{m}} \sum_{K^{\prime} \in \Omega_{m}} u_{h}\right|_{K^{\prime}}\left(\boldsymbol{x}_{m}\right) & \text { if } m \in \mathcal{N}_{\gamma, k} \backslash \mathcal{N}_{\gamma, k}^{\partial},\end{cases}
$$

and $\# \Omega_{m}$ denotes the number of elements of $\mathcal{T}_{h}$ contained in the patch $\Omega_{m}$.

3.5.3. Computable error bounds. The foregoing developments show that each of the quantities

$$
\begin{aligned}
\eta_{C F, K} & =\left\|a^{-1 / 2}\left(\boldsymbol{\sigma}_{h}^{*}-a \nabla u_{h}\right)\right\|_{K}+\left.\frac{h_{K}}{\pi} a\right|_{K} ^{-1 / 2}\left\|f-\Pi_{k-1} f\right\|_{K}, \\
\eta_{N C, K} & =\left\|a^{1 / 2} \nabla\left(u_{h}-u_{h}^{*}\right)\right\|_{K}
\end{aligned}
$$

can be computed directly from the primal HDG approximation using purely local computations. The next result shows that together these quantities provide a computable, constant-free, upper bound on the broken energy seminorm of the error:

Theorem 1. Let $\eta_{C F, K}$ and $\eta_{N C, K}$ be defined as in (26), and let the stabilization parameter $\alpha_{h}$ be of the form (12) where $\gamma$ satisfies (13). Then

$$
\left\|\left(e_{u}, \widehat{e}_{u}\right)\right\|_{p r}^{2} \leq \eta^{2}=\sum_{K \in \mathcal{T}_{h}}\left(\eta_{C F, K}^{2}+\eta_{N C, K}^{2}\right) .
$$

Moreover, there exists a positive constant $c$, depending only on the shape-regularity of the mesh, the polynomial degree $k$, and the local permeability ratio between neighboring elements, such that

$$
c \sum_{K \in \mathcal{T}_{h}}\left(\eta_{C F, K}^{2}+\eta_{N C, K}^{2}\right) \leq\left\|\left(e_{u}, \widehat{e}_{u}\right)\right\|_{p r}^{2}+\sum_{K \in \mathcal{T}_{h}} o s c_{k-1}^{2}(f, K) .
$$

Furthermore,

$$
\left\|\left(e_{u}, \widehat{e}_{u}\right)\right\|_{H D G, p r}^{2} \leq \eta_{H D G}^{2}=\eta^{2}+\sum_{K \in \mathcal{T}_{h}}\left\langle\alpha_{h}\left(P_{M} u_{h}-\widehat{u}_{h}\right), P_{M} u_{h}-\widehat{u}_{h}\right\rangle_{\partial K},
$$

and

$$
c \eta_{H D G}^{2} \leq\left\|\left(e_{u}, \widehat{e}_{u}\right)\right\|_{H D G, p r}^{2}+\sum_{K \in \mathcal{T}_{h}} o s c_{k-1}^{2}(f, K) .
$$

where $c$ is as above. 
The proof of this result is similar to [2, Theorem 2] and an outline of the main steps is given in Section 6 below. Numerical examples illustrating the bounds in practice are given in Section 5 .

\section{The MiXed HDG Methods And COMPUtable ERRor BOUndS}

4.1. The mixed HDG formulation. Whereas the primal HDG method gives an approximation for $\left(u,\left.u\right|_{\mathcal{E}_{h}}\right)$, the mixed HDG method seeks, in addition, to approximate the flux $\left(a \nabla u, u,\left.u\right|_{\mathcal{E}_{h}}\right)$.

Let $\alpha_{h} \in M_{h, 0}^{\text {d.c. }}$ be a positive stabilization parameter to be specified later, and define the bilinear form $\mathcal{B}_{h}^{m x}: \mathbb{X}_{h, k} \times \mathbb{X}_{h, k} \rightarrow \mathbb{R}$ by

$$
\begin{gathered}
\mathcal{B}_{h}^{m x}((\boldsymbol{\sigma}, u, \widehat{u}),(\boldsymbol{\tau}, v, \widehat{v}))=\sum_{K \in \mathcal{T}_{h}}\left\{\left(a^{-1} \boldsymbol{\sigma}, \boldsymbol{\tau}\right)_{K}+(u, \nabla \cdot \boldsymbol{\tau})_{K}-\langle\widehat{u}, \boldsymbol{\tau} \cdot \boldsymbol{n}\rangle_{\partial K}\right. \\
\left.+(\boldsymbol{\sigma}, \nabla v)_{K}-\left\langle\boldsymbol{\sigma} \cdot \boldsymbol{n}-\alpha_{h}(u-\widehat{u}), v-\widehat{v}\right\rangle_{\partial K}\right\},
\end{gathered}
$$

along with the linear form $\mathcal{L}_{h}^{m x}: \mathbb{X}_{h, k} \rightarrow \mathbb{R}$ by

$$
\mathcal{L}_{h}^{m x}((\boldsymbol{\tau}, v, \widehat{v}))=\sum_{K \in \mathcal{T}_{h}}(f, v)_{K}
$$

An approximation of the true solution $\left(a \nabla u, u,\left.u\right|_{\mathcal{E}_{h}}\right)$ is obtained by seeking $\left(\boldsymbol{\sigma}_{h}, u_{h}, \widehat{u}_{h}\right) \in \mathbb{X}_{h, k}$ such that

$$
\mathcal{B}_{h}^{m x}\left(\left(\boldsymbol{\sigma}_{h}, u_{h}, \widehat{u}_{h}\right),\left(\boldsymbol{\tau}_{h}, v_{h}, \widehat{v}_{h}\right)\right)=\mathcal{L}_{h}^{m x}\left(\left(\boldsymbol{\tau}_{h}, v_{h}, \widehat{v}_{h}\right)\right) \quad \forall\left(\boldsymbol{\tau}_{h}, v_{h}, \widehat{v}_{h}\right) \in \mathbb{X}_{h, k} .
$$

This scheme was originally termed the local discontinuous Galerkin-hybridizable method (LDG-H) 22 but is referred to here as the mixed HDG approximation.

4.2. The choice of the stabilization parameter $\alpha_{h}$. The mixed HDG scheme enjoys greater stability properties than the primal HDG scheme as the stabilization parameter $\alpha_{h}$ need only be (partially) positive in order for the scheme to be wellposed as the following result 22, Proposition 3.2] shows.

Lemma 3. If the nonnegative stabilization parameter $\alpha_{h}$ is chosen such that $\alpha_{h}>0$ on at least one facet $F \in \mathcal{E}(K)$ for every element $K$, then there exists a unique solution $\left(\boldsymbol{\sigma}_{h}, u_{h}, \widehat{u}_{h}\right) \in \mathbb{X}_{h, k}$ for $k \geq 0$.

Remark 2 (Stabilization parameter). The two most common choices of stabilization parameter used in practice are:

- uniform stabilization

$$
\left.\alpha_{h}\right|_{F}=\left.a\right|_{K} \quad \text { for all } F \in \mathcal{E}(K), \text { for all } K \in \mathcal{T}_{h},
$$

- single-facet stabilization

$$
\left.\alpha_{h}\right|_{F}=\left\{\begin{array}{ll}
\left.a\right|_{K} & \text { if } F=F_{K}^{*} \\
0 & \text { if } F \neq F_{K}^{*}
\end{array} \quad \text { for all } F \in \mathcal{E}(K), \quad \text { for all } K \in \mathcal{T}_{h},\right.
$$

where $F_{K}^{*}$ is an arbitrarily chosen but fixed facet of $K$.

Each of the above choices of stabilization parameters results in an optimal a priori convergence rate $\mathcal{O}\left(h^{k+1}\right)$ of the error in the energy norm [23]. 
4.2.1. The broken energy seminorm and the $H D G$ energy seminorm. For a given function $(\boldsymbol{\tau}, v, \widehat{v}) \in \mathbb{X}_{h, k, \delta}+\left(H(\operatorname{div} ; \Omega) \times H^{1}(\Omega) \times L^{2}\left(\mathcal{E}_{h}\right)\right)$, let the broken energy seminorm $\|\cdot \cdot\|_{m x}$ be denoted by

$$
\|(\boldsymbol{\tau}, v, \widehat{v})\|_{m x}=\left(\sum_{K \in \mathcal{T}_{h}}\left(a^{-1} \boldsymbol{\tau}, \boldsymbol{\tau}\right)_{K}\right)^{1 / 2},
$$

and let the mixed HDG energy seminorm $\|\cdot\| \|_{H D G, m x}$ be denoted by

$$
\|(\boldsymbol{\tau}, v, \widehat{v}) \mid\|_{H D G, m x}=\left(\|(\boldsymbol{\tau}, v, \widehat{v})\|_{m x}^{2}+\sum_{K \in \mathcal{T}_{h}} h_{K}\left\langle\alpha_{h}(v-\widehat{v}),(v-\widehat{v})\right\rangle_{\partial K}\right)^{1 / 2}
$$

The error in the mixed HDG finite-element approximation is denoted by $\left(\boldsymbol{e}_{\sigma}, e_{u}, \widehat{e}_{u}\right)=$ $\left(\boldsymbol{\sigma}-\boldsymbol{\sigma}_{h}, u-u_{h},\left.u\right|_{\mathcal{E}_{h}}-\widehat{u}_{h}\right)$, where $(\boldsymbol{\sigma}, u)$ is the solution to (1) and $\left(\boldsymbol{\sigma}_{h}, u_{h}, \widehat{u}_{h}\right)$ is the solution to 31 . Similarly to the primal HDG case, we have $e_{u}-\widehat{e}_{u}=-\left(u_{h}-\widehat{u}_{h}\right)$ and hence the quantity

$$
\sum_{K \in \mathcal{T}_{h}} h_{K}\left\langle\alpha_{h}\left(e_{u}-\widehat{e}_{u}\right),\left(e_{u}-\widehat{e}_{u}\right)\right\rangle_{\partial K}
$$

can be evaluated directly given the mixed HDG approximation. Consequently, given a constant free estimator for the broken energy seminorm of the error, we automatically have a constant free estimator for the HDG energy seminorm of the error as well. The next result shows that, by analogy with the primal HDG case, the HDG energy seminorm of the error is equivalent to the broken energy seminorm when the single-facet stabilization $32 \mathrm{~b}$ is used.

However, the equivalence fails to hold if the uniform stabilization $(32 \mathrm{a}$ ) is employed. For instance, in the case of lowest order $(k=0)$ approximation, the discrete energy norm (plus the data oscillation) cannot control the jump term $\sum_{K \in \mathcal{T}_{h}} h_{K}\left\langle\alpha_{h}\left(u_{h}-\widehat{u}_{h}\right),\left(u_{h}-\widehat{u}_{h}\right)\right\rangle_{\partial K}$, as shown by the counterexample constructed in [24, Section 2.4.1]. The situation for general $k \in \mathbb{N}$ remains an open problem at this time.

Lemma 4. Let the stabilization parameter $\alpha_{h}$ given by $32 \mathrm{~b}$, then the HDG energy seminorm and the broken energy seminorm of the error $\left(\boldsymbol{e}_{\sigma}, e_{u}, \widehat{e}_{u}\right)=\left(\boldsymbol{\sigma}-\boldsymbol{\sigma}_{h}, u-\right.$ $\left.u_{h},\left.u\right|_{\mathcal{E}_{h}}-\widehat{u}_{h}\right)$ are equivalent in the sense that

$$
\left\|\left(\boldsymbol{e}_{\sigma}, e_{u}, \widehat{e}_{u}\right)\right\|_{m x} \leq\left\|\left(\boldsymbol{e}_{\sigma}, e_{u}, \widehat{e}_{u}\right)\right\|_{H D G, m x},
$$

and there exists a positive constant $c$, depending only on the shape-regularity of the mesh and the polynomial degree $k$, such that

$$
c\left\|\left(\boldsymbol{e}_{\sigma}, e_{u}, \widehat{e}_{u}\right)\right\|_{H D G, m x}^{2} \leq\left\|\left(\boldsymbol{e}_{\sigma}, e_{u}, \widehat{e}_{u}\right)\right\|_{m x}^{2}+\sum_{K \in \mathcal{T}_{h}}{ }_{\operatorname{osc}}^{2}(f, K) .
$$

Proof. The result follows at once from Lemma 7 in Section 6 below.

4.3. Local conservation. Similarly to the primal HDG scheme (11), the mixed HDG scheme (31) is locally conservative but this time in the sense that the numerical flux

satisfies

$$
\widehat{\boldsymbol{\sigma}}_{h, m x}:=\boldsymbol{\sigma}_{h}-\alpha_{h}\left(u_{h}-\widehat{u}_{h}\right) \boldsymbol{n} \in\left[M_{h, k}^{\mathrm{dc}}\right]^{d}
$$

$$
(f, 1)_{K}+\left\langle\widehat{\boldsymbol{\sigma}}_{h, m x} \cdot \boldsymbol{n}, 1\right\rangle_{\partial K}=0 \quad \text { for each element } K \in \mathcal{T}_{h}
$$


along with

$$
\left.\llbracket \widehat{\boldsymbol{\sigma}}_{h, m x} \rrbracket\right|_{F}=0 \quad \text { on each interior facet } F \in \mathcal{E}_{h}^{o} .
$$

4.4. The computable error bounds. We are now in a position to present computable error bounds for the discrete energy error. While the basic approach is motivated by the technique used in [3,5,27, for the mixed methods, the post-processing technique needed for the mixed HDG case is quite different.

4.4.1. Local (equilibrated) flux post-processing. Let $\boldsymbol{\sigma}_{h}^{*} \in \Sigma_{h, k+1}$ satisfy the following conditions, on each element $K$ :

$$
\begin{aligned}
\left(\nabla \cdot \boldsymbol{\sigma}_{h}^{*}, v\right)_{K} & =-(f, v)_{K} & & \forall v \in \mathbb{P}_{k}(K) \text { and }(v, 1)_{K}=0, \\
\left\langle\boldsymbol{\sigma}_{h}^{*} \cdot \boldsymbol{n}, \widehat{v}\right\rangle_{\partial K} & =\left\langle\widehat{\boldsymbol{\sigma}}_{h, m x} \cdot \boldsymbol{n}, \widehat{v}\right\rangle_{F} & & \forall \widehat{v} \in \mathbb{P}_{k+1}(F), \quad \forall F \in \mathcal{E}(K), \\
\left(\boldsymbol{\sigma}_{h}^{*}, \boldsymbol{\tau}\right)_{K} & =\left(\boldsymbol{\sigma}_{h}, \boldsymbol{\tau}\right)_{K} & & \forall \boldsymbol{\tau} \in \Sigma_{k+1, s b b},
\end{aligned}
$$

where the divergence-free "bubble" space $\Sigma_{k+1, s b b}$ is defined as in 23). Thanks to the local conservation properties 20 and $(21)$, we conclude that $\boldsymbol{\sigma}_{h}^{*} \in \Sigma_{h, k+1} \cap$ $H(\operatorname{div} ; \Omega)$ satisfies

$$
\nabla \cdot \boldsymbol{\sigma}_{h}^{*}=-\Pi_{k} f \text { on } \mathcal{T}_{h} \text {, and } \boldsymbol{\sigma}_{h}^{*} \cdot \boldsymbol{n}=\widehat{\boldsymbol{\sigma}}_{h, m x} \cdot \boldsymbol{n} \text { on } \partial \mathcal{T}_{h} .
$$

4.4.2. Local potential post-processing. A global continuous potential is constructed by averaging a higher order discontinuous approximation to the potential. However, the averaging scheme is more involved than the one used in the primal case: Firstly, we find $u_{h}^{* \text { dc }} \in V_{h, k+1}$ so that, on each element $K$, there holds

$$
\begin{aligned}
\left(a \nabla u_{h}^{*, \mathrm{dc}}, \nabla v\right)_{K} & =\left(\boldsymbol{\sigma}_{h}, \nabla v\right)_{K} \quad \forall v \in \mathbb{P}_{k+1}(K), \\
\left(u_{h}^{*, \mathrm{dc}}, 1\right)_{K} & =\left(u_{h}, 1\right)_{K},
\end{aligned}
$$

The continuous potential post-processing $u_{h}^{*}=S_{k+1}\left(u_{h}^{*, \mathrm{dc}}\right) \in V_{h, k+1} \cap H^{1}(\Omega)$ is then a simple averaging of the degrees of freedom for $u_{h}^{*, \text { dc }}$, where $S_{k+1}(\cdot)$ is defined as in (25).

4.4.3. Computable error bounds. Each of the quantities

$$
\begin{aligned}
\eta_{C F, K} & =\left\|a^{-1 / 2}\left(\boldsymbol{\sigma}_{h}^{*}-\boldsymbol{\sigma}_{h}\right)\right\|_{K}+\left.\frac{h_{K}}{\pi} a\right|_{K} ^{-1 / 2}\left\|f-\Pi_{k} f\right\|_{K}, \\
\eta_{N C, K} & =\left\|a^{-1 / 2}\left(\boldsymbol{\sigma}_{h}-a \nabla u_{h}^{*}\right)\right\|_{K},
\end{aligned}
$$

can be computed directly from the mixed HDG approximation using only local computations. These quantities provide computable, constant-free, upper bounds on the the broken energy seminorm of the error $\left(\boldsymbol{e}_{\sigma}, e_{u}, \widehat{e}_{u}\right)$ :

Theorem 2. Let $\eta_{C F, K}$ and $\eta_{N C, K}$ be defined as in (26), with the stabilization parameter $\alpha_{h}$ chosen to be either (32a) or (32b). Then

$$
\left\|\left(\boldsymbol{e}_{\sigma}, e_{u}, \widehat{e}_{u}\right)\right\|_{m x}^{2} \leq \eta^{2}=\sum_{K \in \mathcal{T}_{h}}\left(\eta_{C F, K}^{2}+\eta_{N C, K}^{2}\right) .
$$


Moreover, there exists a positive constant c, depending only on the shape-regularity of the mesh, the polynomial degree $k$, and the local permeability ratio between neighboring elements, such that

$$
\begin{aligned}
c \sum_{K \in \mathcal{T}_{h}}\left(\eta_{C F, K}^{2}+\eta_{N C, K}^{2}\right) \leq\left\|\left(\boldsymbol{e}_{\sigma}, e_{u}, \widehat{e}_{u}\right)\right\|_{m x}^{2} & \\
& \quad+\sum_{K \in \mathcal{T}_{h}}\left(h_{K}\left\langle\alpha_{h}\left(u_{h}-\widehat{u}_{h}\right), u_{h}-\widehat{u}_{h}\right\rangle_{\partial K \backslash F_{K}^{*}}+o s c_{k}^{2}(f, K)\right),
\end{aligned}
$$

where $F_{K}^{*}$ is an arbitrary but fixed facet of $K$. Furthermore,

$$
\left\|\left(\boldsymbol{e}_{\sigma}, e_{u}, \widehat{e}_{u}\right)\right\|_{H D G, m x}^{2} \leq \eta_{H D G}^{2}=\eta^{2}+\sum_{K \in \mathcal{T}_{h}} h_{K}\left\langle\alpha_{h}\left(u_{h}-\widehat{u}_{h}\right), u_{h}-\widehat{u}_{h}\right\rangle_{\partial K}
$$

with

$$
c \eta_{H D G}^{2} \leq\left\|\left(e_{u}, \widehat{e}_{u}\right)\right\|_{H D G, m x}^{2}+\sum_{K \in \mathcal{T}_{h}} o s c_{k}^{2}(f, K) .
$$

The proof of Theorem 2 is presented in Section 6 .

Remark 3 (Single-facet stabilization). If the stabilization parameter is chosen as in $(32 \mathrm{~b})$, then we can take $F_{K}^{*} \in \mathcal{E}(K)$ in 43 to be the unique facet on which $\alpha_{h}$ is non-zero which implies that

$$
\sum_{K \in \mathcal{T}_{h}} h_{K}\left\langle\alpha_{h}\left(u_{h}-\widehat{u}_{h}\right), u_{h}-\widehat{u}_{h}\right\rangle_{\partial K \backslash F_{K}^{*}}=0 .
$$

Consequently, for the choice (32b), the estimator $\eta^{2}$ also gives a lower bound for the broken energy seminorm.

\section{NumericAl EXAMPLES}

In order to illustrate the results in Theorem 112, we consider Poisson problems in two and three dimensions approximated using the primal and mixed HDG schemes (11) and (31). The implementation is performed using the Python interface of the NGSolve software [46 47]. Conveniently, NGSolve provides a set of basis functions for the divergence-free bubble space $\Sigma_{k, s b b}$ [23] [45] which makes the implementation of the equilibrated flux reconstructions 22 and 39 ) relatively straightforward.

We choose the stabilization parameter for the primal HDG schemes (11) to be

$$
\left.\alpha_{h}\right|_{F}=10 k^{2} h_{F}^{-1} \quad \forall F \in \mathcal{E}_{h}
$$

which, thanks to Lemma 1 . ensures well-posedness on shape-regular meshes. We adopt a shorthand notation and denote the primal HDG scheme (11) used in conjunction with the approximation space $\mathbb{V}_{h, k, 0}$ as pr- $\mathrm{P}_{k}$, while if with the approximation space $\mathbb{V}_{h, k, 1}$ used we use the notation: pr-Pred ${ }_{k}^{\text {red }}$ Likewise, we denote the mixed HDG scheme (31) used in conjunction with uniform stabilization (32a) as $m x-\mathrm{P}_{k}-\mathrm{U}$, while if the single-facet stabilization $32 \mathrm{~b}$ ) is used, we write mx- ${ }_{k}-\mathrm{S}$. In all cases we use static condensation whereby the local, cell-wise, degrees of freedom are eliminated leaving only those degrees of freedom which are located on the mesh skeleton.

We take polynomial degree $k \in\{1,2,3,4\}$ for the first example, and $k \in\{1,2,3\}$ for the second example. 
5.1. Example 1: Two-dimensional L-shaped problem. Here we consider the Laplace problem on a planar L-shaped domain $\Omega_{2 D}=(-1,1) \times(0,1) \cup(-1,0) \times$ $(-1,0]$ with Dirichlet boundary conditions. The initial mesh is shown in Fig. 1(A). The true solution is given by $u(r, \theta)=r^{2 / 3} \sin (2 \theta / 3)$ in polar coordinates.

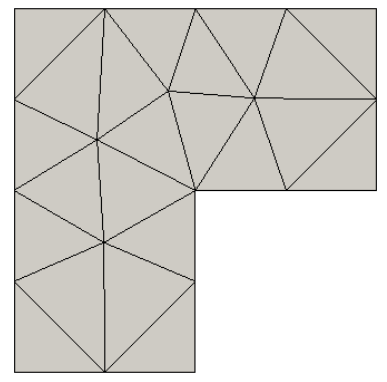

(A) Example 1

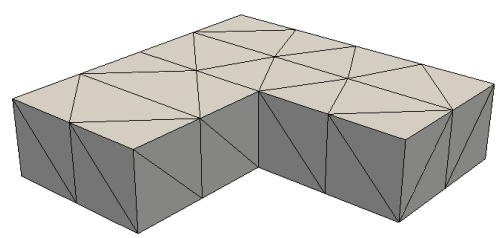

(в) Example 2

FIgURE 1. Initial meshes used in numerical examples.

The sequence of meshes was constructed by selecting for refinement the smallest number of elements whose combined contribution toward the estimator of the broken energy seminorm of the error exceeds half of the total estimated error. A sample of the meshes for the pr- $\mathrm{P}_{k}$ scheme with $k=1$ and $k=4$ is shown in Fig. 2 .
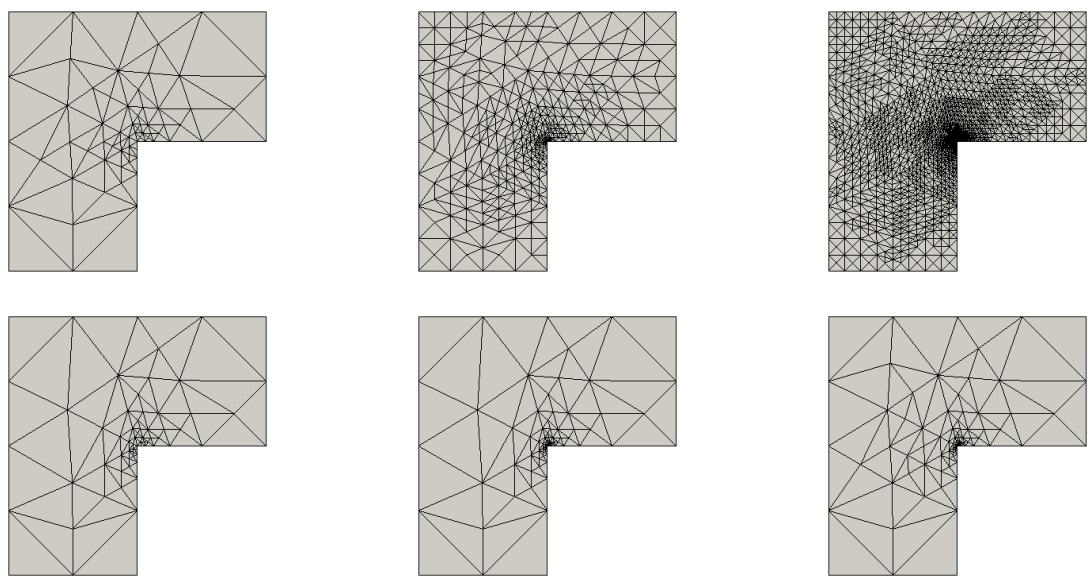

FIGURE 2. The 4th, 9th and 13th meshes obtained performing adaptive refinement for Example 1. Top: $\operatorname{pr}^{-\mathrm{P}_{k}}$ scheme with $k=1$; Bottom: pr- $\mathrm{P}_{k}$ scheme with $k=4$.

In Fig. 3, we plot the error for the primal scheme in the broken energy seminorm (14) against the total number of degrees of freedom $\operatorname{dim} \mathbb{V}_{h, k, \delta}$ and the number of 
global degrees of freedom, $\operatorname{dim} M_{h, k-\delta}^{0}$, remaining after local variables have been eliminated. The error in the broken energy seminorm (33) for the mixed HDG schemes is shown in Fig. 4 In all cases, the effectivity indices are found to lie in
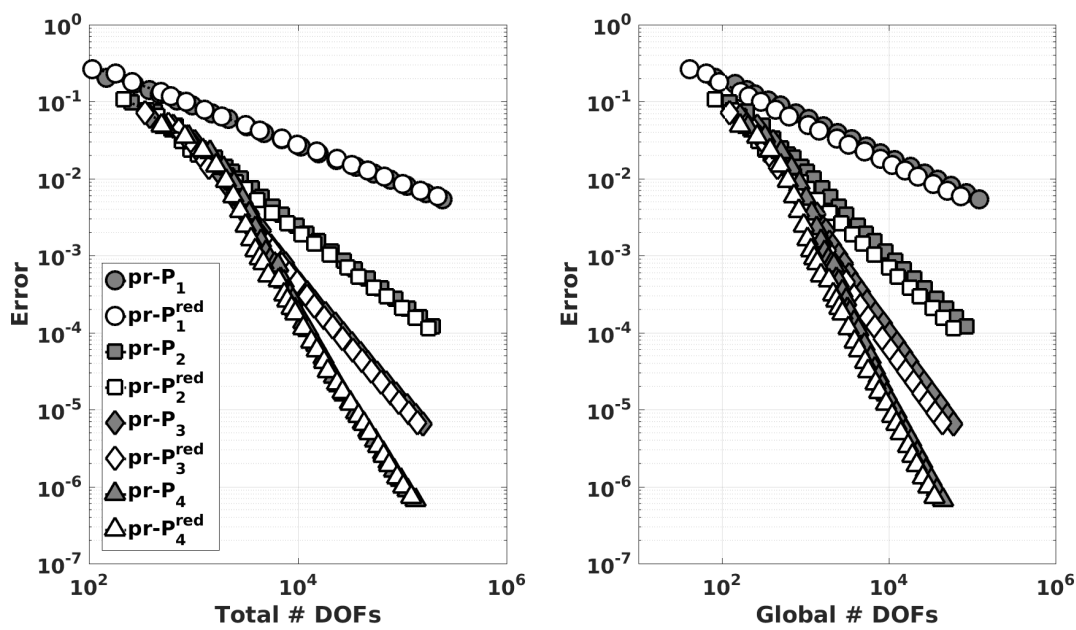

FiguRE 3. Convergence history of the broken energy seminorm error for primal HDG schemes. Left: error against total number of DOFs; Right: error against number of global skeleton DOFs.
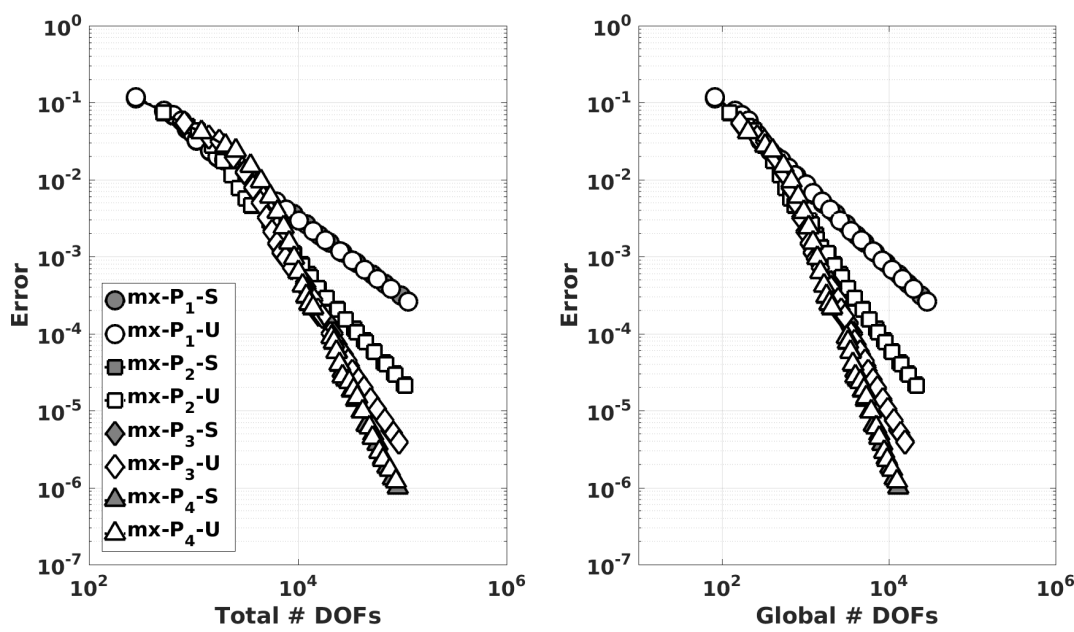

Figure 4. Convergence history of the broken energy seminorm error for mixed HDG schemes. Left: error against total number of DOFs; Right: error against number of global skeleton DOFs.

the range 1.0-3.0 as shown in Fig 5 . 

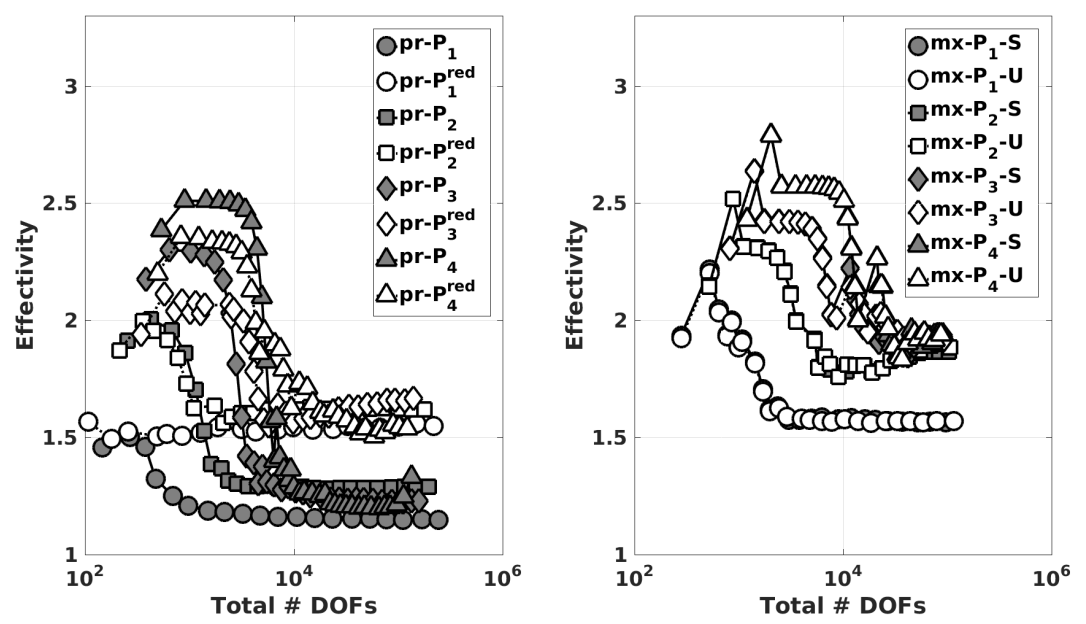

FiguRE 5. History of effectivity indices $\eta /$ error. Left: primal HDG schemes; Right: mixed HDG schemes.

5.2. Example 2: Three-dimensional L-shaped problem. Here we consider the Laplace problem on a three-dimensional L-shaped domain $\Omega_{3 D}=\Omega_{2 D} \times(0,0.5)$, where $\Omega_{2 D}$ is the two-dimensional L-shaped domain in the previous example. The initial mesh is shown in Fig. 1(B). The true solution is independent of $z$, and reduces to the same two-dimensional solution as Example 1. However, the fact that the true solution is independent of $z$ is not used in the finite element analysis and the meshes are unstructured through the thickness.

A sample of the meshes obtained for adaptive solution using the pr- $\mathrm{P}_{k}$ scheme with $k=1$ and $k=3$ is shown in Fig. 6 The errors for the primal and mixed HDG schemes are plotted in Fig. 7 and Fig. 8 , respectively. As before, the effectivity indices are found to vary in the range 1.0-3.0 as shown in Fig. 9

\section{Proofs}

We now turn to the proofs of the results.

As remarked earlier, the jump term in the HDG energy (semi)norm is directly computable, and, as such, we need only concern ourselves with obtaining estimates for the broken energy seminorm of the error. To this end, recall the following Helmholtz decomposition 28,36]:

Lemma 5. Let $\Omega$ be a simply connected polygon/polyhedron. Then, any $\boldsymbol{\tau} \in$ $L^{2}(\Omega)^{d}, d \in\{2,3\}$, can be written in the form

$$
\boldsymbol{\tau}=a \nabla \phi+\nabla \times \psi,
$$

where $\phi \in H_{0}^{1}(\Omega)$ satisfies

$$
(a \nabla \phi, \nabla v)=(\boldsymbol{\tau}, \nabla v) \quad \forall v \in H_{0}^{1}(\Omega) .
$$

and $\psi \in H^{1}(\Omega)^{2 d-3}$ satisfies

$$
\left(a^{-1} \nabla \times \psi, \nabla \times \psi\right)=\left(a^{-1} \boldsymbol{\tau}, \nabla \times \psi\right) .
$$



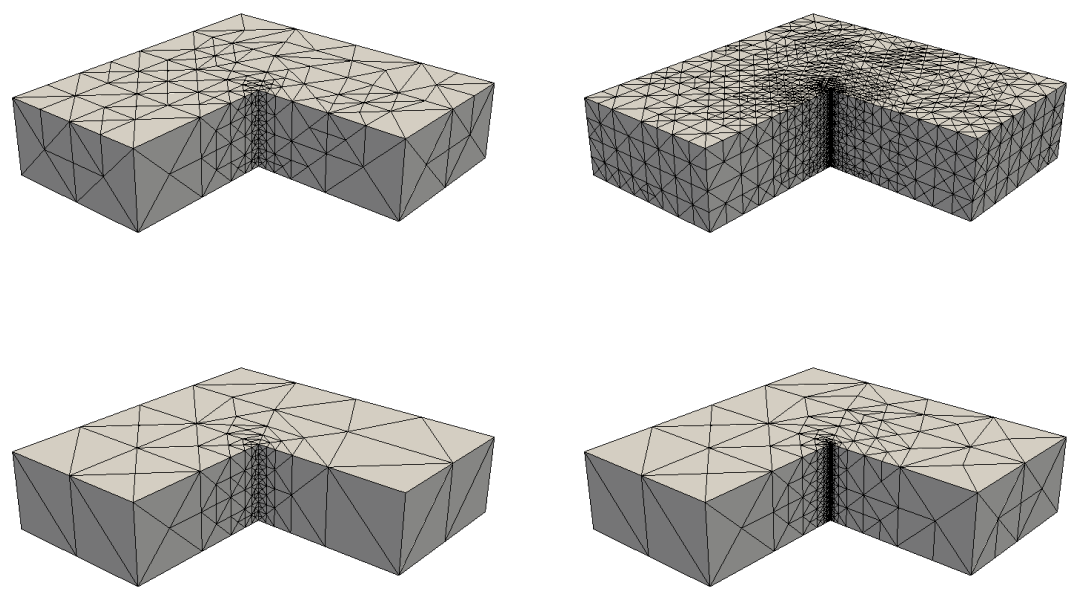

FiguRE 6 . The 4 th and 7 th adaptively refined meshes for Example 2. Top: $\operatorname{pr}^{-\mathrm{P}_{k}}$ scheme with $k=1$; Bottom: $\operatorname{pr}^{-\mathrm{P}_{k}}$ scheme with $k=3$.
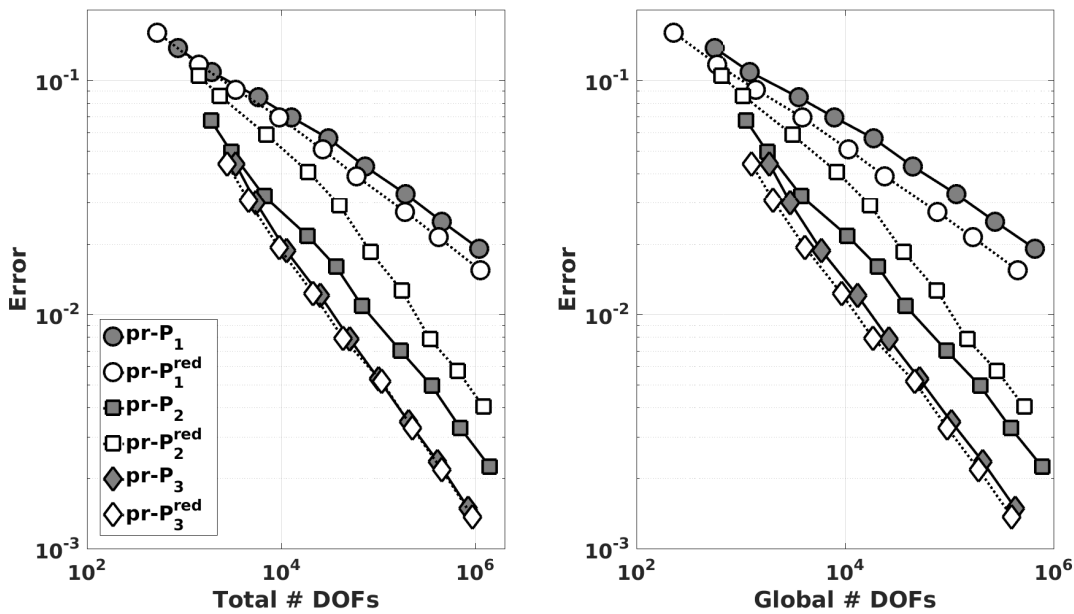

Figure 7. Convergence history of the broken energy seminorm error for primal HDG schemes. Left: error against total number of DOFs; Right: error against number of global DOFs.

Moreover, the decomposition is orthogonal

$$
\left\|a^{-1 / 2} \boldsymbol{\tau}\right\|^{2}=\left\|a^{1 / 2} \nabla \phi\right\|^{2}+\left\|a^{-1 / 2} \nabla \times \psi\right\|^{2} .
$$



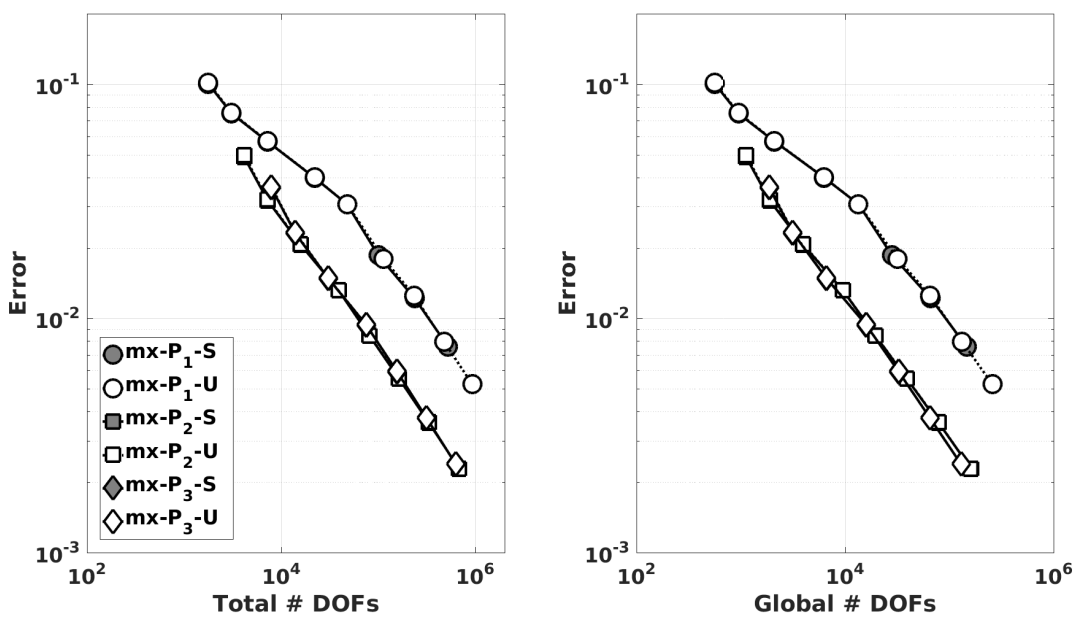

FiguRE 8. Convergence history of the broken energy seminorm error for mixed HDG schemes. Left: error against total number of DOFs; Right: error against number of global DOFs.
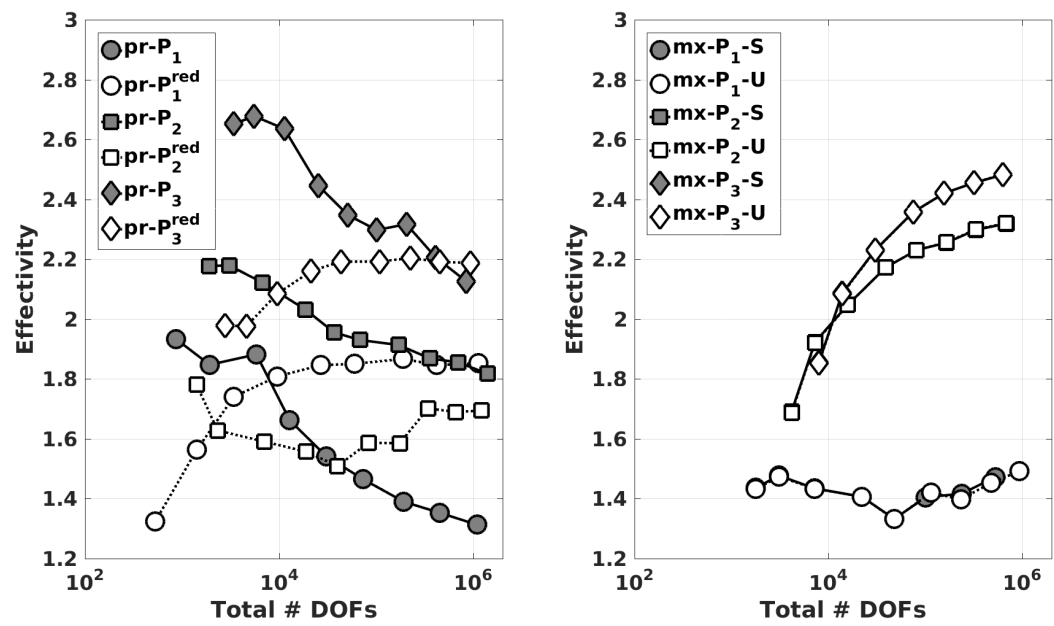

FiguRE 9. History of effectivity indices $\eta$ /error. Left: primal HDG schemes; Right: mixed HDG schemes.

We shall use the decomposition (45) in conjunction with $\tau$ defined elementwise by $a \nabla u-a \nabla u_{h}$ for the primal HDG scheme (11), or by $a \nabla u-\sigma_{h}$ for the mixed HDG scheme (31). In both cases, Lemma 5 gives an orthogonal decomposition of the broken energy seminorm error into the sum of a conforming part $\left\|a^{1 / 2} \nabla \phi\right\|^{2}$ and a nonconforming part $\left\|a^{-1 / 2} \nabla \times \psi\right\|^{2}$. It then suffices to obtain an a posteriori error bound for each part separately and sum to obtain an estimator for the total error. 
6.1. Proof of Theorem 1. The proof of Theorem 1 follows from 10 for the symmetric interior penalty discontinuous Galerkin methods almost verbatim. Specifically, the upper bound (27) in Theorem 1 follows from $49 \mathrm{a}$ and $49 \mathrm{~b}$ below, and the lower bound (28) follows from Lemma 2, (49c) and $449 \mathrm{~d})$.

The following estimates follows from results in 10, Lemma 6.2-6.5] using the proof in 9 , Section 6]. Let $\phi$ and $\psi$ be taken as in (45) in the case where $\boldsymbol{\tau}=$ $a \nabla u-a \nabla u_{h}$, and let $\eta_{C F, K}$ and $\eta_{N C, K}$ be given by (26). Then,

$$
\begin{aligned}
\left\|a^{1 / 2} \nabla \phi\right\|^{2} & \leq \sum_{K \in \mathcal{T}_{h}} \eta_{C F, K}^{2} \\
\left\|a^{-1 / 2} \nabla \times \psi\right\|^{2} & \leq \sum_{K \in \mathcal{T}_{h}} \eta_{N C, K}^{2}
\end{aligned}
$$

Moreover, there exists a positive constant $c$, depending only on the shape-regularity of the mesh and the polynomial degree $k$, such that

$$
\begin{aligned}
c \eta_{C F, K}^{2} \leq & a h_{K}^{-1}\left\|P_{M}\left(u_{h}-\widehat{u}_{h}\right)\right\|_{\partial K}^{2} \\
& +\left\|a^{1 / 2} \nabla \phi\right\|_{K}^{2}+o s c_{k-1}^{2}(f, K), \\
c \eta_{N C, K}^{2} \leq & \sum_{F \in \widetilde{\mathcal{E}}_{K}} a h_{F}^{-1}\left\|\llbracket u_{h} \rrbracket\right\|_{F}^{2},
\end{aligned}
$$

where $\widetilde{\mathcal{E}}_{K}=\left\{F \in \partial \mathcal{T}_{h}: \bar{F} \cap \bar{K}\right.$ is nonempty $\}$.

6.2. Proof of Theorem 2, Theorem 2 is a consequence of the following three lemmas:

Lemma 6. Let $\phi$ and $\psi$ be given by in the decomposition (45), where $\boldsymbol{\tau}$ is chosen to be $\boldsymbol{\tau}=a \nabla u-\boldsymbol{\sigma}_{h}$, and let $\eta_{C F, K}$ and $\eta_{N C, K}$ be given by (42). Then,

$$
\begin{aligned}
\left\|a^{1 / 2} \nabla \phi\right\|^{2} & \leq \sum_{K \in \mathcal{T}_{h}} \eta_{C F, K}^{2} \\
\left\|a^{-1 / 2} \nabla \times \psi\right\|^{2} & \leq \sum_{K \in \mathcal{T}_{h}} \eta_{N C, K}^{2}
\end{aligned}
$$

Moreover, there exists a positive constant $c$, depending only on the shape-regularity of the mesh and the polynomial degree $k$, such that

$$
\begin{aligned}
c \eta_{C F, K}^{2} \leq & h_{K}\left\langle\alpha_{h}\left(u_{h}-\widehat{u}_{h}\right), u_{h}-\widehat{u}_{h}\right\rangle_{\partial K} \\
& +\left\|a^{1 / 2} \nabla \phi\right\|_{K}^{2}+o s c_{k}^{2}(f, K) \\
c \eta_{N C, K}^{2} \leq & \left.a\right|_{K}\left(\left\|\nabla u_{h}^{*, \mathrm{dc}}-a^{-1} \boldsymbol{\sigma}_{h}\right\|_{K}^{2}+\sum_{F \in \widetilde{\mathcal{E}}_{K}} h_{F}^{-1}\left\|\llbracket u_{h}^{*, \mathrm{dc}} \rrbracket\right\|_{F}^{2}\right)
\end{aligned}
$$

where $\widetilde{\mathcal{E}}_{K}=\left\{F \in \partial \mathcal{T}_{h}: \bar{F} \cap \bar{K}\right.$ is nonempty $\}$. 
Proof. Direct computation gives

$$
\begin{aligned}
& \left\|a^{1 / 2} \nabla \phi\right\|^{2}=\left(a \nabla u-\sigma_{h}, \nabla \phi\right) \\
& =(f, \phi)-\left(\boldsymbol{\sigma}_{h}, \nabla \phi\right) \quad \text { (integration by parts) } \\
& =\left(f-\Pi_{k} f, \phi\right)+\left(\Pi_{k} f, \phi\right) \\
& +\sum_{K \in \mathcal{T}_{h}}\left\langle\widehat{\boldsymbol{\sigma}}_{h, m x} \cdot \boldsymbol{n}, \phi\right\rangle_{\partial K}-\left(\boldsymbol{\sigma}_{h}, \nabla \phi\right) \quad(\text { conservation 38) } \\
& =\left(f-\Pi_{k} f, \phi\right)+\left(\boldsymbol{\sigma}_{h}^{*}-\boldsymbol{\sigma}_{h}, \nabla \phi\right) \quad \text { (equilibration 40p) } \\
& \leq \eta_{C F, K}\left\|a^{1 / 2} \nabla \phi\right\|,
\end{aligned}
$$

where the last inequality follows from the Cauchy-Schwarz and the Poincaré inequalities. This completes the proof of (50a). Turning to $(50 \mathrm{~b})$, since $(\nabla v, \nabla \times \psi)=0$ for all $v \in H_{0}^{1}(\Omega)$, we have

$$
\begin{aligned}
\left\|a^{-1 / 2} \nabla \times \psi\right\|^{2} & =\left(\nabla u-a^{-1} \boldsymbol{\sigma}_{h}, \nabla \times \psi\right)=\left(\nabla u_{h}^{*}-a^{-1} \boldsymbol{\sigma}_{h}, \nabla \times \psi\right) \\
& \leq \eta_{N C, K}\left\|a^{-1 / 2} \nabla \times \psi\right\| .
\end{aligned}
$$

Let $\boldsymbol{\rho}_{h}=\boldsymbol{\sigma}_{h}^{*}-\boldsymbol{\sigma}_{h}$, then for all $K \in \mathcal{T}_{h}$, by (39), we have $\boldsymbol{\rho}_{h} \in \mathbb{P}_{k+1}(K)^{d}$ satisfies

$$
\begin{aligned}
\left(\nabla \cdot \boldsymbol{\rho}_{h}, v\right)_{K} & =-\left(f+\nabla \cdot \boldsymbol{\sigma}_{h}, v\right)_{K} & & \forall v \in \mathbb{P}_{k}(K) \text { and }(v, 1)_{K}=0, \\
\left\langle\boldsymbol{\rho}_{h} \cdot \boldsymbol{n}, \widehat{v}\right\rangle_{\partial K} & =-\left\langle\alpha_{h}\left(u_{h}-\widehat{u}_{h}\right), \widehat{v}\right\rangle_{F} & & \forall \widehat{v} \in \mathbb{P}_{k+1}(F), \quad \forall F \in \mathcal{E}(K), \\
\left(\boldsymbol{\rho}_{h}, \boldsymbol{\tau}\right)_{K} & =0 & & \forall \boldsymbol{\tau} \in \Sigma_{k+1, s b b} .
\end{aligned}
$$

Hence, there exists a constant $c$, depending only on the polynomial degree $k$ and shape-regularity of the element $K$, such that

$$
c\left\|\rho_{h}\right\|_{K}^{2} \leq h_{K}^{2}\left\|f+\nabla \cdot \sigma_{h}\right\|_{K}^{2}+h_{K}\left\|\alpha_{h}\left(u_{h}-\widehat{u}_{h}\right)\right\|_{\partial K}^{2},
$$

while, using a standard bubble function technique 7,50, we have

$$
c h_{K}^{2}\left\|f+\nabla \cdot \boldsymbol{\sigma}_{h}\right\|_{K}^{2} \leq\|a \nabla \phi\|_{K}^{2}+\left.a\right|_{K} \operatorname{osc}_{k}^{2}(f, K) .
$$

The choice of stabilization parameter 32 means that

$$
\left\|\alpha_{h}\left(u_{h}-\widehat{u}_{h}\right)\right\|_{\partial K}^{2}=\left.a\right|_{K}\left\|\alpha_{h}^{1 / 2}\left(u_{h}-\widehat{u}_{h}\right)\right\|_{\partial K}^{2},
$$

and the proof of (50c) follows from these estimates.

Finally, we have

$$
\begin{aligned}
& \left\|\nabla u_{h}^{*}-a^{-1} \boldsymbol{\sigma}_{h}\right\|_{K}^{2} \leq 2\left(\left\|\nabla u_{h}^{*}-\nabla u_{h}^{*, \mathrm{dc}}\right\|_{K}^{2}+\left\|\nabla u_{h}^{*, \mathrm{dc}}-a^{-1} \boldsymbol{\sigma}_{h}\right\|_{K}^{2}\right), \\
& \left\|\nabla u_{h}^{*}-\nabla u_{h}^{*, \mathrm{dc}}\right\|_{K}^{2} \leq c \sum_{F \in \widetilde{\mathcal{E}}_{K}} h_{F}^{-1}\left\|\llbracket u_{h}^{*, \mathrm{dc}} \rrbracket\right\|_{F}^{2} .
\end{aligned}
$$

Combining the above estimates completes the proof of (50d).

Lemma 7. There exists a positive constant , depending only on the shape-regularity of the mesh and the polynomial degree $k$, such that for any facet $F \in \mathcal{E}(K)$,

$$
\begin{aligned}
c h_{K}\left\|\alpha_{h}^{1 / 2}\left(u_{h}-\widehat{u}_{h}\right)\right\|_{F}^{2} \leq & h_{K}\left\|\alpha_{h}^{1 / 2}\left(u_{h}-\widehat{u}_{h}\right)\right\|_{\partial K \backslash F}^{2} \\
& +\left\|a^{1 / 2} \nabla \phi\right\|_{K}^{2}+o s c_{k}^{2}(f, K) .
\end{aligned}
$$


Proof. The mixed HDG scheme (31) satisfies, for every $K \in \mathcal{T}_{h}$,

$$
\left\langle\alpha_{h}\left(u_{h}-\widehat{u}_{h}\right), v\right\rangle_{\partial K}=\left(f+\nabla \cdot \boldsymbol{\sigma}_{h}, v\right)_{K} \quad \text { for all } v \in \mathbb{P}_{k}(K) .
$$

Let the function $z \in \mathbb{P}_{k}(K)$ satisfy

$$
\begin{aligned}
(z, w)_{K} & =0 \quad \text { for all } w \in \mathbb{P}_{k-1}(K), \\
\langle z, \widehat{w}\rangle_{F^{*}} & =\left\langle\alpha_{h}\left(u_{h}-\widehat{u}_{h}\right), \widehat{w}\right\rangle_{F^{*}} \quad \text { for all } w \in \mathbb{P}_{k}\left(F^{*}\right),
\end{aligned}
$$

where $F^{*}$ is a fixed facet of $K$. We have, by a standard scaling argument,

$$
\|z\|_{K} \leq c h_{K}^{1 / 2}\left\|\alpha_{h}\left(u_{h}-\widehat{u}_{h}\right)\right\|_{F^{*}} .
$$

Taking $v=z$ in (54) and rearranging terms, we obtain

$$
\begin{aligned}
&\left\|\alpha_{h}\left(u_{h}-\widehat{u}_{h}\right)\right\|_{F^{*}}^{2}=\left(f+\nabla \cdot \boldsymbol{\sigma}_{h}, z\right)_{K}+\left\langle\alpha_{h}\left(u_{h}-\widehat{u}_{h}\right), z\right\rangle_{\partial K \backslash F^{*}} \\
& \leq c\left(\left\|f+\nabla \cdot \boldsymbol{\sigma}_{h}\right\|_{K}+h_{K}^{-1 / 2}\left\|\alpha_{h}\left(u_{h}-\widehat{u}_{h}\right)\right\|_{\partial K \backslash F^{*}}\right)\|z\|_{K} \\
& \leq c\left(h_{K}^{1 / 2}\left\|f+\nabla \cdot \boldsymbol{\sigma}_{h}\right\|_{K}+\left\|\alpha_{h}\left(u_{h}-\widehat{u}_{h}\right)\right\|_{\partial K \backslash F^{*}}\right)\left\|\alpha_{h}\left(u_{h}-\widehat{u}_{h}\right)\right\|_{F^{*}}
\end{aligned}
$$

The proof is completed by invoking estimate $(52)$ for the cell-wise residual term $f+\nabla \cdot \boldsymbol{\sigma}_{h}$

Lemma 8. There exists a positive constant c, depending only on the shape-regularity of the mesh and the polynomial degree $k$, such that

$$
\begin{aligned}
c\left\|\nabla u_{h}^{*, \mathrm{dc}}-a^{-1} \boldsymbol{\sigma}_{h}\right\|_{K}^{2} & \leq\left\|\nabla u-a^{-1} \boldsymbol{\sigma}_{h}\right\|_{K}^{2}, \\
c h_{F}^{-1}\left\|\llbracket u_{h}^{*, \mathrm{dc}} \rrbracket\right\|_{F}^{2} & \leq \sum_{K^{\prime} \in \widetilde{F}}\left\|\nabla u-a^{-1} \boldsymbol{\sigma}_{h}\right\|_{K^{\prime}}^{2} .
\end{aligned}
$$

Proof. We first prove the estimate (55). We denote $\boldsymbol{\rho}_{h}=\nabla u_{h}^{*, \mathrm{dc}}-a^{-1} \boldsymbol{\sigma}_{h}$ and let the function $\boldsymbol{\rho}_{h}^{*} \in \mathbb{P}_{k+1}(K)^{d} \oplus \boldsymbol{x} \widetilde{\mathbb{P}}_{k+1}(K)$ be defined as follows:

$$
\begin{aligned}
& \left\langle\boldsymbol{\rho}_{h}^{*} \cdot \boldsymbol{n}, \widehat{v}\right\rangle_{F}=0 \quad \forall \widehat{v} \in \mathbb{P}_{k+1}(F), \quad \forall F \in \mathcal{E}(K), \\
& \left(\boldsymbol{\rho}_{h}^{*}, \boldsymbol{v}\right)_{K}=\left(\boldsymbol{\rho}_{h}, \boldsymbol{v}\right)_{K} \quad \forall \boldsymbol{v} \in \mathbb{P}_{k}(K)^{d},
\end{aligned}
$$

then, we have

$$
c\left\|\boldsymbol{\rho}_{h}^{*}\right\|_{K} \leq\left\|\boldsymbol{\rho}_{h}\right\|_{K} .
$$

Moreover, by equations (41a) and (57), we have

$$
\left(\nabla \cdot \boldsymbol{\rho}_{h}^{*}, v\right)_{K}=-\left(\boldsymbol{\rho}_{h}^{*}, \nabla v\right)_{K}=-\left(\boldsymbol{\rho}_{h}, \nabla v\right)_{K}=0 \quad \text { for all } v \in \mathbb{P}_{k+1}(K) .
$$

This implies that $\nabla \cdot \boldsymbol{\rho}_{h}^{*}=0$ because $\nabla \cdot \boldsymbol{\rho}_{h}^{*} \in \mathbb{P}_{k+1}(K)$. Since $\boldsymbol{\rho}_{h}^{*}$ has vanishing normal trace on $\partial K$ by equations (57a), we obtain

$$
\left(\boldsymbol{\rho}_{h}^{*}, \nabla v\right)_{K}=0 \quad \text { for all } v \in H^{1}(K)
$$

Hence,

$$
\left\|\boldsymbol{\rho}_{h}\right\|_{K}^{2}=\left(\boldsymbol{\rho}_{h}^{*}, \boldsymbol{\rho}_{h}\right)_{K}=\left(\boldsymbol{\rho}_{h}^{*}, \nabla u_{h}^{*, \mathrm{dc}}-a^{-1} \boldsymbol{\sigma}_{h}\right)_{K}=\left(\boldsymbol{\rho}_{h}^{*}, \nabla u-a^{-1} \boldsymbol{\sigma}_{h}\right)_{K}
$$

The estimate 55 now follows from the Cauchy-Schwarz inequality. 
Let $P_{M_{0}}$ be the $L^{2}$-projection onto the space $\mathbb{P}_{0}(F)$. Applying the results in 27 . Lemma 3.4-3.5], we obtain

$$
\begin{aligned}
h_{F}^{-1}\left\|\llbracket u_{h}^{*, \mathrm{dc}} \rrbracket\right\|_{F}^{2} & =h_{F}^{-1}\left\|P_{M_{0}} \llbracket u_{h}^{*, \mathrm{dc}} \rrbracket\right\|_{F}^{2}+h_{F}^{-1}\left\|\left(\mathrm{Id}-P_{M_{0}}\right) \llbracket u_{h}^{*, \mathrm{dc}} \rrbracket\right\|_{F}^{2} \\
& \leq c \sum_{K \in \widetilde{F}}\left(\left\|a^{-1} \boldsymbol{\sigma}_{h}-\nabla u_{h}^{*, \mathrm{dc}}\right\|_{K}^{2}+\left\|\nabla u-\nabla u_{h}^{*, \mathrm{dc}}\right\|_{K}^{2}\right) .
\end{aligned}
$$

The estimate (56) now immediately follows from the triangle inequality and (55).

6.3. Proof of Lemma 1. Since $\mathbb{V}_{h, k, \delta}$ is a finite-dimensional space, it suffices to show that $\left(u_{h}, \widehat{u}_{h}\right)=(0,0)$ is the only solution to the homogeneous problem. Let

$$
C_{k, K}:=\sum_{F^{\prime} \in \mathcal{E}(K)} \frac{\left|F^{\prime}\right|^{2}}{|K|}, \text { and } D_{k, K}:=\frac{k(k+d-1)}{d} C_{k, K},
$$

then for $\left(v_{h}, \widehat{v}_{h}\right) \in \mathbb{V}_{h, k, \delta}$, we have

$$
\begin{aligned}
\mathcal{B}_{h}^{p r}\left(\left(v_{h}, \widehat{v}_{h}\right),\left(v_{h}, \widehat{v}_{h}\right)\right)= & \sum_{K \in \mathcal{T}_{h}}\left\{\left(a \nabla v_{h}, \nabla v_{h}\right)_{K}-2\left\langle a \nabla v_{h} \cdot \boldsymbol{n}, v_{h}-\widehat{v}_{h}\right\rangle_{\partial K}\right. \\
& \left.+\left\langle\frac{a \gamma C_{k, K}}{|F|}\left(P_{M} v_{h}-\widehat{v}_{h}\right), P_{M} v_{h}-\widehat{v}_{h}\right\rangle_{\partial K}\right\},
\end{aligned}
$$

Since $\left.a \nabla v_{h} \cdot \boldsymbol{n}\right|_{F} \in \mathbb{P}_{k-1}(F)$, there holds

$$
\left\langle a \nabla v_{h} \cdot \boldsymbol{n}, v_{h}-\widehat{v}_{h}\right\rangle_{\partial K}=\left\langle a \nabla v_{h} \cdot \boldsymbol{n}, P_{M} v_{h}-\widehat{v}_{h}\right\rangle_{\partial K} .
$$

By the Cauchy-Schwarz and Young's inequalities, for any $\epsilon>0$,

$$
\begin{aligned}
2\left\langle a \nabla v_{h} \cdot \boldsymbol{n}, v_{h}\right. & \left.-\widehat{v}_{h}\right\rangle_{F} \leq \frac{|F|}{a \epsilon}\left\|a \nabla v_{h} \cdot \boldsymbol{n}\right\|_{F}^{2}+\frac{a \epsilon}{|F|}\left\|P_{M} v_{h}-\widehat{v}_{h}\right\|_{F}^{2} \\
& \leq \frac{|F|}{a \epsilon} \frac{k(k+d-1)}{d} \frac{|F|}{|K|}\left\|a \nabla v_{h}\right\|_{K}^{2}+\frac{a \epsilon}{|F|}\left\|P_{M} v_{h}-\widehat{v}_{h}\right\|_{F}^{2}
\end{aligned}
$$

where, the final inequality holds thanks to the inverse-trace inequality $[52]$ and $\left.a \nabla u\right|_{K} \in \mathbb{P}_{k-1}(K)^{d}$. Summing the above inequality over $F \in \mathcal{E}(K)$ gives

$$
2\left\langle a \nabla v_{h} \cdot \boldsymbol{n}, v_{h}-\widehat{v}_{h}\right\rangle_{\partial K} \leq \frac{D_{k, K}}{\epsilon}\left\|a^{1 / 2} \nabla v_{h}\right\|_{K}^{2}+\sum_{F \in \mathcal{E}(K)} \frac{a \epsilon}{|F|}\left\|P_{M} v_{h}-\widehat{v}_{h}\right\|_{F}^{2} .
$$

Hence, we have

$$
\begin{aligned}
\mathcal{B}_{h}^{p r}\left(\left(v_{h}, \widehat{v}_{h}\right),\left(v_{h}, \widehat{v}_{h}\right)\right) \geq & \sum_{K \in \mathcal{T}_{h}}\left(1-\frac{D_{k, K}}{\epsilon}\left\|a^{1 / 2} \nabla v_{h}\right\|_{K}^{2}\right. \\
& \left.+\sum_{F \in \mathcal{E}(K)} \frac{a\left(\gamma C_{k, K}-\epsilon\right)}{|F|}\left\|P_{M} v_{h}-\widehat{v}_{h}\right\|_{F}^{2}\right) .
\end{aligned}
$$

Finally, if $\gamma$ satisfies 13 then there exists an $\epsilon$ such that

$$
\gamma C_{k, K}>\epsilon>D_{k, K} \text {. }
$$

Consequently, when the right hand side of (11) vanishes, $\left\|\nabla v_{h}\right\|_{K}=0$ for all $K \in \mathcal{T}_{h}$ and $\left\|P_{M} v_{h}-\widehat{v}_{h}\right\|_{F}=0$ for all $F \in \mathcal{E}_{h}$. Hence, the only solution to the homogeneous problem is $\left(v_{h}, \widehat{v}_{h}\right)=(0,0)$, and therefore there exists a unique solution $\left(u_{h}, \widehat{u}_{h}\right) \in \mathbb{V}_{h, k, \delta}$ to 11. 
6.4. Proof of Lemma 2, The proof follows that of 2, Theorem 3].

By the conservation property (21), we have

$$
\left.\widehat{u}_{h}\right|_{F}= \begin{cases}0 & \text { if } F \in \mathcal{E}_{h}^{\partial}, \\ \frac{\left\{\alpha_{h} P_{M} u_{h}\right\}}{\left\{\alpha_{h}\right\}}-\frac{1}{2\left\{\alpha_{h}\right\}} \llbracket a \nabla u_{h} \rrbracket & \text { if } F \in \mathcal{E}_{h}^{o} .\end{cases}
$$

Hence,

$$
P_{M} u_{h}-\left.\widehat{u}_{h}\right|_{F}= \begin{cases}P_{M} u_{h} & \text { if } F \in \mathcal{E}_{h}^{\partial}, \\ \frac{\llbracket P_{M} u_{h} \rrbracket \cdot n}{2 \alpha_{h}\left\{1 / \alpha_{h}\right\}}+\frac{1}{2\left\{\alpha_{h}\right\}} \llbracket a \nabla u_{h} \rrbracket & \text { if } F \in \mathcal{E}_{h}^{o} .\end{cases}
$$

Inserting the above expression into the jump term in the HDG energy norm and regrouping gives

$$
\begin{aligned}
\sum_{K \in \mathcal{T}_{h}}\left\langle\alpha_{h} P_{M}\left(u_{h}-\widehat{u}_{h}\right), P_{M}\left(u_{h}-\widehat{u}_{h}\right)\right\rangle_{\partial K} & \\
=\sum_{F \in \mathcal{E}_{h}^{o}} & \left(\left\langle\frac{1}{2\left\{1 / \alpha_{h}\right\}} \llbracket P_{M} u_{h} \rrbracket, \llbracket P_{M} u_{h} \rrbracket\right\rangle_{F}+\left\langle\frac{1}{2\left\{\alpha_{h}\right\}} \llbracket a \nabla u_{h} \rrbracket, \llbracket a \nabla u_{h} \rrbracket\right\rangle_{F}\right) \\
& +\sum_{F \in \mathcal{E}_{h}^{\partial}}\left\langle\alpha_{h} \llbracket P_{M} u_{h} \rrbracket, \llbracket P_{M} u_{h} \rrbracket\right\rangle_{F}
\end{aligned}
$$

Here, the gradient jump term can be controlled by the standard bubble function technique 7,50

$$
c \sum_{F \in \mathcal{E}_{h}^{o}} h_{F}\left\|\llbracket a \nabla u_{h} \rrbracket\right\|_{F}^{2} \leq\left\|\left(e_{u}, \widehat{e}_{u}\right)\right\|_{p r}^{2}+\sum_{K \in \mathcal{T}_{h}} \operatorname{osc}_{k-1}^{2}(f, K) .
$$

On the other hand, there holds

$$
\left\|\llbracket P_{M} u_{h} \rrbracket\right\|_{F}^{2}=|F|\left(\overline{\llbracket P_{M} u_{h} \rrbracket}\right)^{2}+\left\|\llbracket P_{M} u_{h} \rrbracket-\overline{\llbracket P_{M} u_{h} \rrbracket}\right\|_{F}^{2},
$$

where $\overline{\llbracket P_{M} u_{h} \rrbracket}$ denote the average value of $\llbracket P_{M} u_{h} \rrbracket$ on the facet $F$. Thanks to the trace and Poincaré inequalites, we have

$$
c h_{F}^{-1}\left\|\llbracket P_{M} u_{h} \rrbracket-\overline{\llbracket P_{M} u_{h} \rrbracket}\right\|_{F}^{2} \leq \sum_{K^{\prime} \in \widetilde{F}}\left\|\nabla u-\nabla u_{h}\right\|_{K^{\prime}}^{2} .
$$

Hence, to show norm equivalence, it remains to show that the term

$$
\sum_{F \in \mathcal{E}_{h}} \frac{|F|}{h_{F}}\left(\overline{\llbracket P_{M} u_{h} \rrbracket}\right)^{2}
$$

can be controlled by the discrete energy seminorm plus the data oscillation. Replacing $\widehat{u}_{h}$ in the primal HDG scheme (11) with the expression (59), we obtain

$$
\begin{aligned}
0= & \left(f-\nabla \cdot a \nabla u_{h}, v_{h}\right)_{K} \\
& -\sum_{F \in \mathcal{E}^{\circ}(K)}\left\langle\frac{\llbracket P_{M} u_{h} \rrbracket \cdot \boldsymbol{n}}{2 \alpha_{h}\left\{1 / \alpha_{h}\right\}}+\frac{1}{2\left\{\alpha_{h}\right\}} \llbracket a \nabla u_{h} \rrbracket, a \nabla v_{h} \cdot \boldsymbol{n}-\alpha_{h} P_{M} v_{h}\right\rangle_{F} \\
& -\sum_{F \in \mathcal{E}^{\partial}(K)}\left\langle P_{M} u_{h}, a \nabla v_{h} \cdot \boldsymbol{n}-\alpha_{h} P_{M} v_{h}\right\rangle_{F}
\end{aligned}
$$


for all $v_{h} \in V_{h, k}$ and $K \in \mathcal{T}_{h}$. Moreover, there holds 49

$$
(d+1)^{2} \rho\left(\boldsymbol{S}_{K}\right) \leq \sum_{F^{\prime} \in \mathcal{E}(K)} \frac{\left|F^{\prime}\right|^{2}}{|K|},
$$

where $\boldsymbol{S}_{K}$ is the element stiffness matrix, i.e. $\boldsymbol{S}_{i j}=\left(\nabla \lambda_{i}, \nabla \lambda_{j}\right)_{K}$ with $\left\{\lambda_{\ell}\right\}_{\ell=1}^{d+1}$ being the barycentric coordinates for the element $K$, and $\rho\left(\boldsymbol{S}_{K}\right)$ is its spectral radius. Hence the stabilization parameter $\alpha_{h}$ in $(12)$, with $\gamma$ satisfying $(13)$, satisfies

$$
\left.\alpha_{h}\right|_{F}>\frac{\left.a\right|_{K}}{|F|}(d+1)^{2} \rho\left(\boldsymbol{S}_{K}\right), \quad \forall F \in \mathcal{E}(K)
$$

The proof is then concluded following [2, Theorem 3] by taking special linear test functions in the equation 60 and using the above estimate for the stabilization parameter.

\section{REFERENCES}

[1] M. Ainsworth, Robust a posteriori error estimation for nonconforming finite element approximation, SIAM J. Numer. Anal., 42 (2005), pp. 2320-2341.

[2] — A posteriori error estimation for discontinuous Galerkin finite element approximation, SIAM J. Numer. Anal., 45 (2007), pp. 1777-1798.

[3] — A posteriori error estimation for lowest order Raviart-Thomas mixed finite elements, SIAM J. Sci. Comput., 30 (2007/08), pp. 189-204.

[4] - A framework for obtaining guaranteed error bounds for finite element approximations, J. Comput. Appl. Math., 234 (2010), pp. 2618-2632.

[5] M. Ainsworth AND X. MA, Non-uniform order mixed FEM approximation: implementation, post-processing, computable error bound and adaptivity, J. Comput. Phys., 231 (2012), pp. 436-453.

[6] M. Ainsworth and J. T. Oden, A unified approach to a posteriori error estimation using element residual methods, Numer. Math., 65 (1993), pp. 23-50.

[7] — A posteriori error estimation in finite element analysis, Pure and Applied Mathematics (New York), Wiley-Interscience [John Wiley \& Sons], New York, 2000.

[8] M. Ainsworth AND R. Rankin, Fully computable bounds for the error in nonconforming finite element approximations of arbitrary order on triangular elements, SIAM J. Numer. Anal., 46 (2008), pp. 3207-3232.

[9] - Fully computable error bounds for discontinuous Galerkin finite element approximations on meshes with an arbitrary number of levels of hanging nodes, SIAM J. Numer. Anal., 47 (2010), pp. 4112-4141.

[10] — Constant free error bounds for nonuniform order discontinuous Galerkin finiteelement approximation on locally refined meshes with hanging nodes, IMA J. Numer. Anal., 31 (2011), pp. 254-280.

[11] M. Ainsworth And R. Rankin, Technical note: A note on the selection of the penalty parameter for discontinuous Galerkin finite element schemes, Numer. Methods Partial Differential Equations, 28 (2012), pp. 1099-1104.

[12] D. N. Arnold, F. Brezzi, B. Cockburn, and L. D. Marini, Unified analysis of discontinuous Galerkin methods for elliptic problems, SIAM J. Numer. Anal., 39 (2001/02), pp. 17491779 .

[13] D. Boffi, F. Brezzi, And M. Fortin, Mixed finite element methods and applications, vol. 44 of Springer Series in Computational Mathematics, Springer, Heidelberg, 2013.

[14] D. Braess, V. Pillwein, And J. SchöBerl, Equilibrated residual error estimates are probust, Comput. Methods Appl. Mech. Engrg., 198 (2009), pp. 1189-1197.

[15] Z. Cai And S. Zhang, Robust equilibrated residual error estimator for diffusion problems: conforming elements, SIAM J. Numer. Anal., 50 (2012), pp. 151-170.

[16] C. Carstensen, M. Eigel, R. H. W. Hoppe, and C. Löbhard, A review of unified a posteriori finite element error control, Numer. Math. Theory Methods Appl., 5 (2012), pp. 509-558. 
[17] C. Carstensen, R. H. W. Hoppe, N. Sharma, and T. Warburton, Adaptive hybridized interior penalty discontinuous Galerkin methods for H(curl)-elliptic problems, Numer. Math. Theory Methods Appl., 4 (2011), pp. 13-37.

[18] C. Carstensen and C. Merdon, Estimator competition for Poisson problems, J. Comput. Math., 28 (2010), pp. 309-330.

[19] - Computational survey on a posteriori error estimators for nonconforming finite element methods for the Poisson problem, J. Comput. Appl. Math., 249 (2013), pp. 74-94.

[20] H. Chen, J. LI, AND W. QIU, Robust a posteriori error estimates for HDG method for convection-diffusion equations, IMA J. Numer. Anal., 36 (2016), pp. 437-462.

[21] S. Cochez-DHONDT AND S. NicAise, Equilibrated error estimators for discontinuous Galerkin methods, Numer. Methods Partial Differential Equations, 24 (2008), pp. 1236-1252.

[22] B. Cockburn, J. Gopalakrishnan, and R. Lazarov, Unified hybridization of discontinuous Galerkin, mixed and continuous Galerkin methods for second order elliptic problems, SIAM J. Numer. Anal., 47 (2009), pp. 1319-1365.

[23] B. Cockburn, J. Gopalakrishnan, and F.-J. Sayas, A projection-based error analysis of HDG methods, Math. Comp., 79 (2010), pp. 1351-1367.

[24] B. Cockburn, R. H. Nochetto, AND W. Zhang, Contraction property of adaptive hybridizable discontinuous Galerkin methods, Math. Comp., 85 (2016), pp. 1113-1141.

[25] B. Cockburn and W. Zhang, A posteriori error estimates for HDG methods, J. Sci. Comput., 51 (2012), pp. 582-607.

[26] — A posteriori error analysis for hybridizable discontinuous Galerkin methods for second order elliptic problems, SIAM J. Numer. Anal., 51 (2013), pp. 676-693.

[27] — An a posteriori error estimate for the variable-degree Raviart-Thomas method, Math. Comp., 83 (2014), pp. 1063-1082.

[28] E. DARI, R. DuRAn, C. PADRA, AND V. VAMPa, A posteriori error estimators for nonconforming finite element methods, RAIRO Modél. Math. Anal. Numér., 30 (1996), pp. 385-400.

[29] P. Destuynder And B. MÉTIVEt, Explicit error bounds for a nonconforming finite element method, SIAM J. Numer. Anal., 35 (1998), pp. 2099-2115.

[30] - Explicit error bounds in a conforming finite element method, Math. Comp., 68 (1999), pp. 1379-1396.

[31] H. Egger And C. Waluga, hp analysis of a hybrid DG method for Stokes flow, IMA J. Numer. Anal., 33 (2013), pp. 687-721.

[32] Y. Epshteyn AND B. RiviÈRe, Estimation of penalty parameters for symmetric interior penalty Galerkin methods, J. Comput. Appl. Math., 206 (2007), pp. 843-872.

[33] A. ERn And M. Vohralí K, Four closely related equilibrated flux reconstructions for nonconforming finite elements, C. R. Math. Acad. Sci. Paris, 351 (2013), pp. 77-80.

[34] - Polynomial-degree-robust a posteriori estimates in a unified setting for conforming, nonconforming, discontinuous Galerkin, and mixed discretizations, SIAM J. Numer. Anal., 53 (2015), pp. 1058-1081.

[35] G. N. Gatica and F. A. Sequeira, A priori and a posteriori error analyses of an augmented HDG method for a class of quasi-Newtonian Stokes flows, J. Sci. Comput., 69 (2016), pp. $1192-1250$.

[36] V. Girault And P.-A. Raviart, Finite element methods for Navier-Stokes equations, Springer-Verlag, Berlin, 1986.

[37] D. W. Kelly, The self-equilibration of residuals and complementary a posteriori error estimates in the finite element method, Internat. J. Numer. Methods Engrg., 20 (1984), pp. 14911506.

[38] K. Y. KIM, A posteriori error analysis for locally conservative mixed methods, Math. Comp., 76 (2007), pp. 43-66.

[39] — A posteriori error estimators for locally conservative methods of nonlinear elliptic problems, Appl. Numer. Math., 57 (2007), pp. 1065-1080.

[40] P. Ladevèze AND D. Leguillon, Error estimate procedure in the finite element method and applications, SIAM J. Numer. Anal., 20 (1983), pp. 485-509.

[41] C. Lehrenfeld, Hybrid Discontinuous Galerkin methods for solving incompressible flow problems, 2010. Diploma Thesis, MathCCES/IGPM, RWTH Aachen.

[42] R. Luce and B. I. Wohlmuth, A local a posteriori error estimator based on equilibrated fluxes, SIAM J. Numer. Anal., 42 (2004), pp. 1394-1414. 
[43] S. Nicaise, K. Witowski, and B. I. Wohlmuth, An a posteriori error estimator for the Lamé equation based on equilibrated fluxes, IMA J. Numer. Anal., 28 (2008), pp. 331-353.

[44] I. OIKaWA, A hybridized discontinuous Galerkin method with reduced stabilization, J. Sci. Comput., 65 (2015), pp. 327-340.

[45] S. ZAGLmayr, High order finite element methods for electromagnetic field computation, 2006. $\mathrm{PhD}$ thesis, Johannes Kepler Universit ät Linz, Linz.

[46] J. SCHÖBERL, NETGEN an advancing front 2d/3d-mesh generator based on abstract rules, Computing and Visualization in Science, 1 (1997), pp. 41-52.

[47] - C++11 Implementation of Finite Elements in NGSolve, 2014. ASC Report 30/2014, Institute for Analysis and Scientific Computing, Vienna University of Technology.

[48] K. Shahbazi, An explicit expression for the penalty parameter of the interior penalty method, Journal of Computational Physics, 205 (2005), pp. 401 - 407.

[49] J. Shewchuк, What is a Good Linear Finite Element? Interpolation, Conditioning, Anisotropy and Quality Measures, Tech. report, Department of Computer Science, University of California, Berkeley, CA, (2003).

[50] R. Verfürth, A review of a posteriori error estimation and adaptive mesh-refinement techniques, Chichester: Wiley-Teubner, 1996.

[51] M. Vohralí K, A posteriori error estimation in the conforming finite element method based on its local conservativity and using local minimization, C. R. Math. Acad. Sci. Paris, 346 (2008), pp. 687-690.

[52] T. Warburton and J. S. Hesthaven, On the constants in hp-finite element trace inverse inequalities, Comput. Methods Appl. Mech. Engrg., 192 (2003), pp. 2765-2773.

Division of Applied Mathematics, Brown University, 182 George St, Providence Ri 02912, USA.

E-mail address: Mark_Ainsworth@brown.edu

Division of Applied Mathematics, Brown University, 182 George St, Providence Ri 02912, USA.

E-mail address: Guosheng_Fu@brown.edu 